\title{
Quasi-optimal Feedback Control for an Isolated Intersection under
}

\author{
Oversaturation \\ Weili Sun ${ }^{\mathrm{a}}$, Yunpeng Wang ${ }^{\mathrm{a}}$, Guizhen Yu ${ }^{\mathrm{a}^{*}}$, and Henry X. Liu ${ }^{\mathrm{b}, \mathrm{c}}$ \\ ${ }^{\text {a }}$ School of Transportation Science and Engineering, Beihang University, Beijing, China \\ ${ }^{b}$ Department of Civil and Environmental Engineering, University of Michigan, Ann Arbor, MI 48109, USA \\ ${ }^{c}$ University of Michigan Transportation Research Institute (UMTRI), Ann Arbor, MI 48109, USA \\ *Corresponding Author: yugz@buaa.edu.cn
}

\begin{abstract}
How to manage signalized intersections under oversaturated conditions is a long-standing problem in traffic science and engineering. However, although research works in this area date back to 1960s, an on-line control strategy with theoretically bounded performance is missing, even for the control of an isolated intersection under oversaturation. This paper makes one step further in this area by proposing a QUEUE-based quasi-optimal feedback control (abbreviated as QUEUE) strategy for an isolated oversaturated intersection. The QUEUE strategy is intuitive, simple, and proved to match the off-line optimum in the case of constant demand. More importantly, the bounds of sub-optimality of the QUEUE strategy can be specified quantitatively in general piece-wise constant demand cases. To better deal with the maximum queue constraints, the oversaturation period is divided into the queuing period and the dissipation period with two different objectives. In the queuing period, the primary objective is to keep the queue length within the maximum value; but for the dissipation period, the primary objective is to eliminate all queues at the earliest time. Interestingly, we found that both control objectives can be realized with the same QUEUE strategy. Numerical examples show that the QUEUE strategy approximates the off-line optimum very well. The average sub-optimality in comparison with the off-line optimum in the challenging conditions with Poisson distributed random demand is below 5\%.
\end{abstract}

Keywords: Oversaturation; isolated intersection; feedback control; quasi-optimal

\section{Introduction}

More and more traffic intersections in cities are operated under oversaturated conditions, especially during the peak hours. Vehicular queues may grow very long due to heavy demands that exceed the maximum discharging capacity of intersections. It will be even more undesirable if vehicle queues spill back to upstream intersections, and then the spillover spreads to more intersections and potentially leads to the gridlock (Daganzo, 2007). Therefore signal control strategies under such conditions must be carefully designed.

Unlike the control of undersaturated intersections for which theories and technical tools are well-established, consensus has not been reached regarding the control policies for oversaturated intersections, even for an isolated oversaturated intersection. For an isolated oversaturated intersection, the main task is to allocate green times among conflicting phases to minimize the duration of oversaturation period as well as the total delay time of all vehicles. Although research literature on optimization of the green splits of an isolated oversaturated intersection dates back to 1960 s, most of them belong to the category of off-line optimization. The pioneer in this field is Gazis (1964). He solved the continuous-time optimal control problem of an oversaturated intersection with two conflicting streams by semi-graphical method, and then proved the optimality of the solution using Pontryagin's maximum principle. The optimal solution is a bang-bang type two-stage strategy. His method minimizes the duration of the oversaturation period firstly, and minimizes the total delay secondly. Based on Gazis' work, Guardabassi et al. (1984) found the necessary conditions for solution existence. Michalopoulos and Stephanopoulos (1977a) took the 
maximum queue constraint into account and offered a numerical method to compute the corresponding optimal solution. Chang and Lin (2000) and Zou et al. (2012) proposed discrete-time optimal control models so as to better match the cycle-by-cycle signal control in the real world. These works are able to find the ideal optimal solution analytically, providing benchmarks for control of an isolated oversaturated intersection. However, these methods rely upon perfect knowledge of OD demand for the whole period, which is very demanding. The same problem exists in the methods dealing with multiple oversaturated intersections (e.g., Michalopoulos and Stephanopoulos, 1977b; Lieberman et al., 2000; Liu and Chang, 2011; Lertworawanich et al., 2011; Park et al., 2000).

One common way of extending these off-line optimal control strategies to on-line strategies is by employing the rolling horizon (model predictive) structure (e.g., Aboudolas et al., 2010). The optimal control problem defined in a short horizon is solved on-line iteratively using the current traffic state as well as predicted demand over the finite horizon. Only the first step of the resultant control actions is implemented, then the traffic state is sampled again and the calculations are repeated starting from the new state and prediction. The rolling-horizon structure and real-time measurement greatly favor the real-world application of off-line strategies. However, prediction of future traffic demand is still required.

An alternative way of on-line traffic control for an oversaturated intersection is the state feedback method. This kind of method directly calculates the value of control variables based on current measured state, not requiring prediction of future demand. To manage an oversaturated intersection, ideally, queue lengths on all directions are needed as state variables. It is not easy to estimate queue lengths in case of oversaturation as the phenomenon of queue over detectors is often encountered (Wu et al., 2010), but the recently developed queue estimation method based on high-resolution data provides a potential solution to the problem (Liu et al., 2009).

Research works about on-line feedback control strategy for an oversaturated intersection are limited. Gordon (1969) developed an algorithm for on-line control of queue lengths at oversaturated intersections to keep the queue length ratios on both roads equal so as to delay spillover as long as possible. Gordon's algorithm is meaningful in case when queues are forming, but not necessary when queues are dissolving in the later period of oversaturation. Ioslovich et al. (2011) concluded in their paper that the optimal control law derived from solving the off-line problem is in a feedback form of current queue lengths. But the conclusion is based on constant arrival rate of traffic and unconstrained queue length. Lin et al. (2011) proposed a queue-based feedback control scheme that maintains the proportion of queue length on one road to another in a desired range. Although several choices of the set point were described as examples and the results are promising, it is still unclear how "good" this control strategy is. The traffic-responsive urban control (TUC) strategy (Diakaki et al., 2002) and the maximum pressure (MP) control strategy (Varaiya, 2013) are also novel feedback control examples that adjust the signal over cycle based on real time measurement of queue lengths. Although not explicit designed to manage oversaturated intersections, the TUC strategy and the MP strategy help to mitigate oversaturation by minimizing (residual) queue length. However, vehicular delay might not be minimized underlying the TUC or MP strategy.

It is essential that reliable performance evaluation of a control strategy is achievable before implementation. Specifically, having the benchmark of off-line optimum in hand, we should investigate quantitatively the sub-optimality of a feedback control strategy for an oversaturated intersection. This allows one to judge the quality of the control strategy. The review of literature shows that such quantitative evaluation is still missing in the existing feedback control strategies for an oversaturation intersection. Questions such as "can the feedback control strategy match the off-line optimum in certain conditions?" and "how far is the performance of feedback control strategy from the off-line optimum in the worst condition?" are not answered yet. Our work is to shed light on this issue, in which a QUEUE-based on-line quasi-optimal feedback control (QUEUE) strategy for an oversaturated intersection is developed and theoretically assessed.

The primary contribution of this paper is that we find an innovative way of approximating the off-line optimal 
control strategy for an oversaturated intersection with an on-line feedback control method. The feedback strategy is based on the availability of detected queue size data, while perfect knowledge or reliable prediction of future demand is not needed. It is proved to match the off-line optimum in case of constant arrival flow rate. More importantly, the upper bounds of the sub-optimality from the off-line optimum are quantified in general cases.

Different from the feedback control models proposed in the past, the QUEUE strategy deal with the maximum queue constraints in a more explicit way. We divide the whole oversaturation period into two parts, namely the queuing period when queues increase continuously and the dissipation period when queues decrease gradually. It is evident that keeping the queue length within the maximum constraints is the primary objective in the queuing period; but in the dissipation period, the primary objective is eliminating all queues at the earliest time, while the maximum queue constraints can be simplified consider or even omitted. Interestingly, we found that the control laws for the two different periods can be developed using the same logic in the QUEUE strategy.

The rest of the paper is organized as follows. Section 2 defines the subject control problem and briefly reviews existing analytical results of off-line optimal control strategy. Section 3 introduces details about the QUEUE strategy, including the division of oversaturation period and the corresponding QUEUE strategy for each period. The optimality of the QUEUE strategy is quantitatively assessed in Section 4, and Section 5 introduces briefly the way of extending the QUEUE strategy to a multi-phase intersection. Numerical examples are employed to show the potential ability of the QUEUE strategy in Section 6. Section 7 concludes this paper and discusses future studies.

\section{Problem description and existing analytical results}

\subsection{Problem description}

Here we focus mainly on developing the QUEUE strategy for an intersection with two-phase signal plans. However, it is shown in Section 5 that the proposed QUEUE strategy can be easily extended to intersections with multi-phase signal plans. In the system of an intersection with two conflicting traffic streams (Fig. 1), dynamic variables for stream $i$ are the inflow demand, queue size (number of queued vehicles) and green time, denoted by $d_{i}$, $n_{i}$ and $g_{i}$, respectively. The main constant parameters are the saturation flow rates at the entrance of the intersection, denoted by $s_{1}$ and $s_{2}$. Note that the term "queue length" rather than "queue size" is more often used in the literature. However, "queue length" may represent different meanings, such as the number of queued vehicles or the distance from the tail of the queue to the head of the queue. To remove any ambiguity, we use "queue size" in the paper to represent the number of queued vehicles.

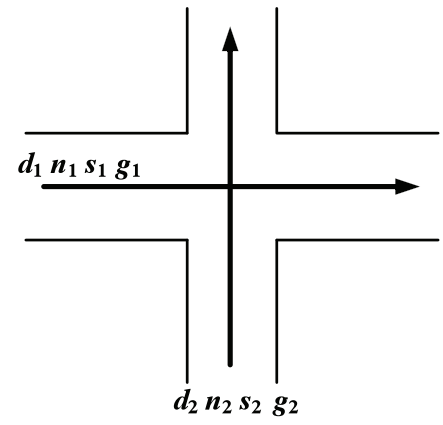

Fig. 1. A traffic intersection with two conflicting traffic streams

The well-known store-and-forward modelling (SFM) of traffic network is employed (Gazis, 1964; Aboudolas et al., 2009). For each traffic stream, the cycle-by-cycle evolution of queue size is expressed by the following conservation equation: 


$$
n_{i}(k+1)=n_{i}(k)+c\left[d_{i}(k)-s_{i} \frac{g_{i}(k)}{c}\right], i=1,2
$$

where $k$ is the index of cycles, $c$ is the common cycle length, a constant.

Oversaturation happens when demand exceeds the maximum service. It is usually defined as follows

$$
\left(d_{1}+n_{1} / c\right) / s_{1}+\left(d_{2}+n_{2} / c\right) / s_{2}>1
$$

Two main reasons, namely the increased demand or the decreased service, can cause oversaturation. The focus of this paper is the oversaturation caused by the increased demand, while the service rates $s_{1}$ and $s_{2}$ are assumed to be constant. Oversaturation caused by the decreased service due to spillover of downstream link will be considered in future work.

Lost time is critical in the decision of optimal cycle length. However, the decision of cycle length is beyond the scope of the paper. Therefore, lost time is not considered here, as it will not change the following discussion except for some notation (Ioslovich, et al., 2011). So, we have

$$
c=g_{1}+g_{2}
$$

As $g_{1}$ and $g_{2}$ are complementary, we can focus on the decision of $g_{1}$, while $g_{2}$ is always readily derived. The green time $g_{1}$ is constrained by a maximum value and a minimum value as follows:

$$
g^{\min } \leq g_{1} \leq g^{\max }
$$

where $g^{\min }$ and $g^{\max }$ are the minimum and maximum green time for road 1, respectively. Here $g^{\min }$ and $g^{\max }$ are not exactly the same with the initial green time and maximum green time (for max out) in the actuated signal control scheme. The values of $g^{\min }$ and $g^{\max }$ could be simply set to the initial green time and maximum green time, but they could also be pre-determined by signal coordination or other considerations.

Lastly, the queue size is restricted by the maximum queue size.

$$
0 \leq n_{i} \leq n_{i}^{\max }
$$

where $n_{i}{ }^{\max }$ denotes the capacity for queued vehicles of approach $i$. This storage capacity is generally defined as the length of the road multiplied by the number of lanes and the jam density. However, the value of $n_{i}{ }^{\text {max }}$ is usually set smaller because the end of the queue may reach upstream intersection before the link is fully jammed. Note again that the values of $n_{i}^{\max }$ can also be predetermined for control purpose.

The problem to be investigated is, finding an optimal trajectory of $\left[g_{1}, g_{2}\right]$ that follows the aforementioned constraints, which makes the oversaturation period ends at the earliest time and the total delay time is minimized as well.

\subsection{Off-line optimal control solution}

The first off-line analytical solution for the above problem was proposed by Gazis (1964). The problem was solved using the semi-graphical method, and the optimality was proved by applying Pontryagin's control theory. Gazis's idea is explained in Fig. 2. The main components in Fig. 2 are the cumulative demand curve (CDC) and the cumulative service curve (CSC). In each approach, the CDC is intrinsically unpredictable and uncontrollable, but assumed a known linear function of time here. The CSC is controlled by signal timing, and is always below CDC during the oversaturation period. The vertical distance between the two curves denotes the current queue size. Note that the maximum queue size constraints are not considered. Without loss of generality, assume that $s_{1}>s_{2}$, then the result of the optimal bang-bang control strategy is shown by a two-stage piecewise linear CSC in Fig. 2. The first stage is giving the major approach the maximum green time (letting $g_{1}=g^{\max }$ ), and the second stage is giving the major approach the minimum green time (letting $g_{1}=g^{\mathrm{min}}$ ). The switch time of the two stages is denoted by $\tau$. The oversaturation period ends at the earliest time $T$, indicated by the time that the CDC and CSC join together simultaneously in both approaches. 


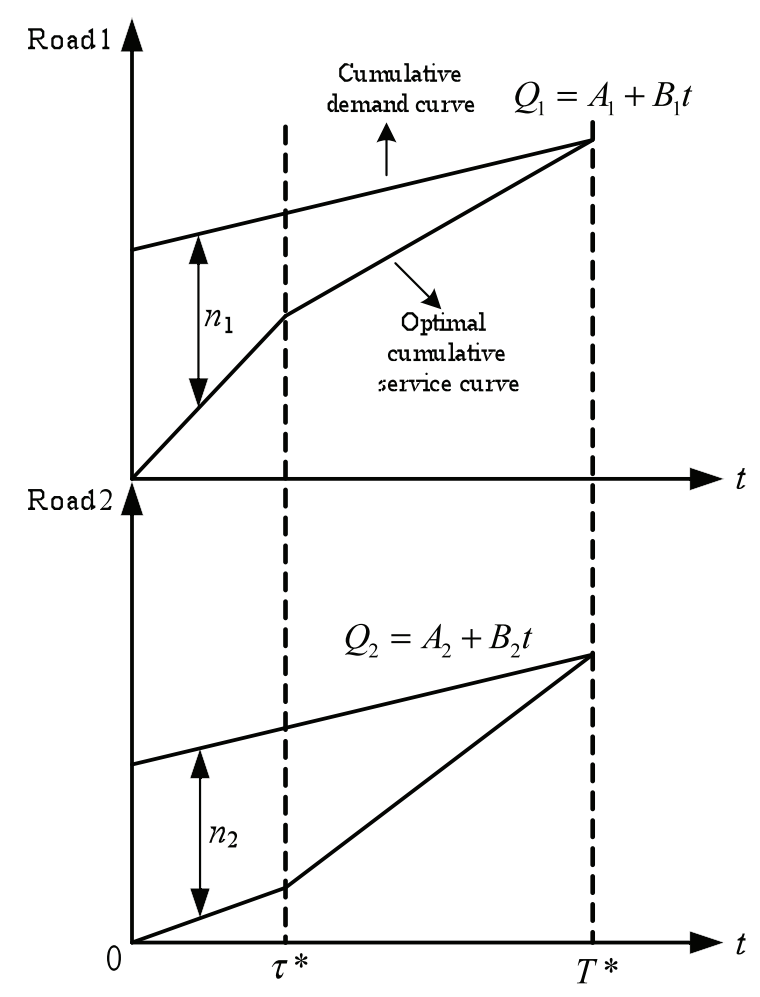

Fig. 2. The two-stage optimal solution for control of an isolated oversaturated intersection

Assume that $n_{1}{ }^{0}=A_{1}$ and $n_{2}{ }^{0}=A_{2}$ at the beginning, and the constant demands are $d_{1} \equiv B_{1}$ and $d_{2} \equiv B_{2}$, then the expression of the CDCs are

$$
Q_{i}=A_{i}+B_{i} t, i=1,2
$$

where $Q_{i}$ is the cumulative demand function of approach $i$.

The optimal values of $\tau$ and $T$ are solved as follows.

$$
\begin{aligned}
& T^{*}=\frac{A_{1} s_{2}+A_{2} s_{1}}{s_{1} s_{2}-B_{1} s_{2}-B_{2} s_{1}} \\
& \tau^{*}=\frac{\left(c / s_{1}\right) Q_{1}(T)-g^{\min } T}{g^{\max }-g^{\min }}=\frac{A_{2} B_{1} c-A_{1} B_{2} c+A_{1} s_{2}\left(c-g^{\min }\right)-A_{2} s_{1} g^{\min }}{\left(s_{1} s_{2}-B_{1} s_{2}-B_{2} s_{1}\right)\left(g^{\max }-g^{\min }\right)} .
\end{aligned}
$$

It is worth noting that the optimal solution shown above minimizes $T$ principally, on which the minimization of total delay time is based. If we relax the requirement that both queues are dissolved simultaneously, as pointed out by Gazis (1964), a further reduction of total delay time may be accomplished by extending the first stage for a proper period.

\subsection{Solution existence condition}

Based on Gazis' work, Guradabassi, et al. (1984) analyzed the existence condition for the optimal solution. In the queue state diagram (Fig. 3), the optimal control problem is to find an optimal trajectory from the initial state $\left[n_{1}{ }^{0}, n_{2}{ }^{0}\right]$ to the origin. With the assumption that the demands are constant (i.e., the CDCs are linear), the maximum and minimum green constraints form two boundary lines from $\left[n_{1}{ }^{0}, n_{2}{ }^{0}\right]$, within which the reachable states region lies. The slopes of the boundary lines are expressed as follows. 


$$
\begin{aligned}
& \left(\frac{\Delta n_{1}}{\Delta n_{2}}\right)^{\max }=\frac{B_{1}-s_{1} g^{\max } / c}{B_{2}-s_{2}\left(1-g^{\max } / c\right)} \\
& \left(\frac{\Delta n_{1}}{\Delta n_{2}}\right)^{\min }=\frac{B_{1}-s_{1} g^{\min } / c}{B_{2}-s_{2}\left(1-g^{\min } / c\right)}
\end{aligned}
$$

where $\Delta n_{1}$ and $\Delta n_{2}$ are queue dissolving rates of road 1 and road 2, respectively; $\left(\Delta n_{1} / \Delta n_{2}\right)^{\max }$ and $\left(\Delta n_{1} / \Delta n_{2}\right)^{\min }$ are the maximum and minimum values of the ratio $\Delta n_{1} / \Delta n_{2}$, respectively.

Consider the objective that requires the earliest end of oversaturation period, queues on both roads should be dissolved completely and simultaneously. In other words, the trajectory in the queue state plane should reach the origin without reaching any axis before. Guardabassi et al. (1984) showed that the two-stage optimal solution exists if and only if the origin belongs to the reachable region.

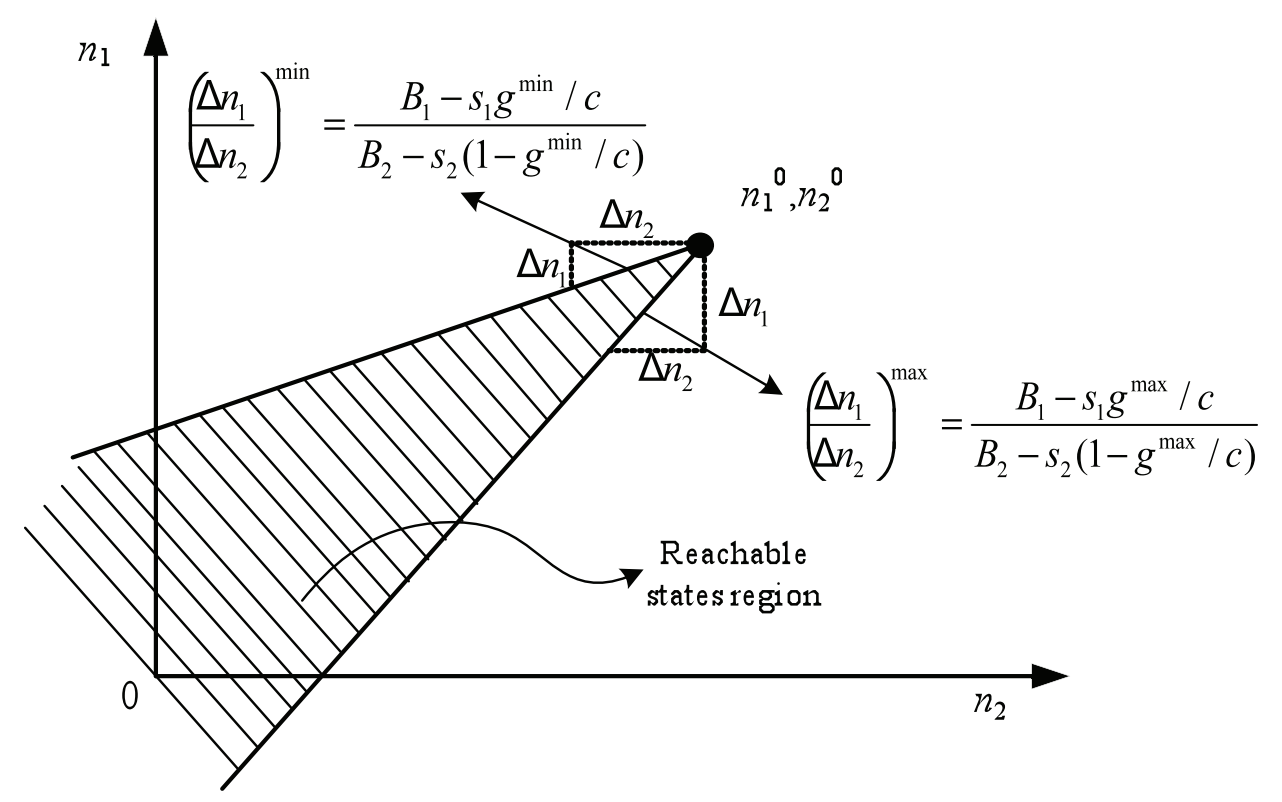

Fig. 3. The queue state diagram

\section{QUEUE-based on-line quasi-optimal feedback control (QUEUE) strategy}

The goal of the proposed control strategy is to approximate the off-line optimal control solution described in the previous section, in an on-line feedback scheme. Instead of calculating simultaneously the entire trajectory of $\left[g_{1}, g_{2}\right]$ for the oversaturation period based on perfect knowledge of OD demand, the QUEUE strategy determine the value of $\left[g_{1}, g_{2}\right]$ every cycle based on traffic flow data. Fig. 4 shows an example of the cycle-by-cycle decision procedure of the QUEUE strategy. At the beginning of the $k+1^{\text {th }}$ cycle, the queue sizes and arrival demands for the past cycle (the $k^{\text {th }}$ cycle) have been detected and stored. Then $\left[g_{1}(k+1), g_{2}(k+1)\right]$ is calculated based on the values of $d_{1}(k), d_{2}(k), n_{1}(k)$ and $n_{2}(k)$. At the end of the $k+1^{\text {th }}$ cycle, the values of $d_{1}(k+1), d_{2}(k+1), n_{1}(k+1)$ and $n_{2}(k+1)$ are ready to be used in the next cycle. Note that $y_{i}(k)=s_{i} g_{i}(k) / c$ is the constant service rate approximation for approach $i$ at the $k^{\text {th }}$ cycle used in the SFM. 


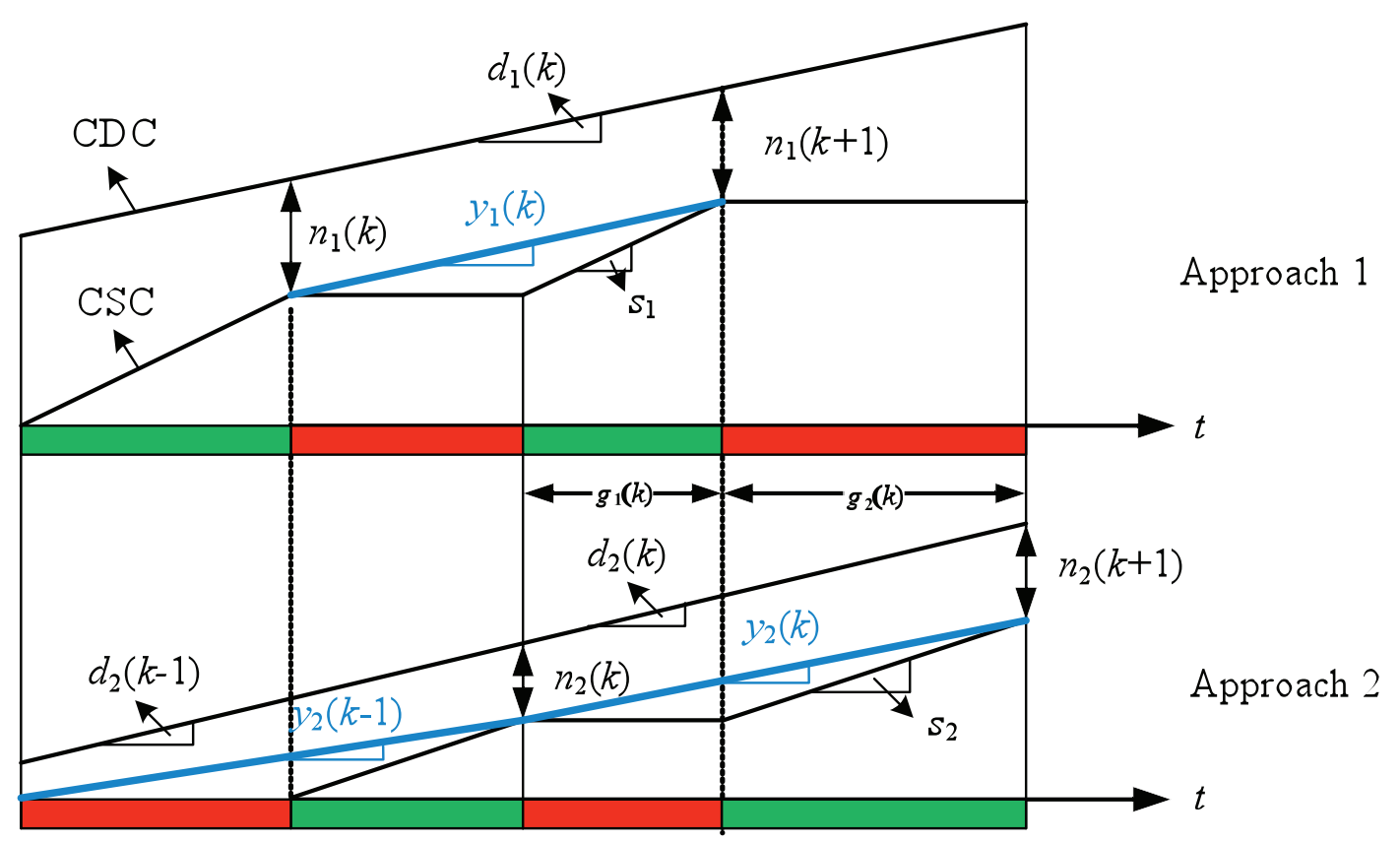

Fig. 4. Cycle-by-cycle evolution of CDCs and CSCs

\subsection{Division of the oversaturation period}

Before going into details of the QUEUE strategy, we should first introduce the special treatment in consideration with the maximum queue constraints. Generally, the evolution of queue sizes has two states during the oversaturation period. The queue sizes increase continuously at first, and then decrease gradually. It is evident that the maximum queue size constraints apply mainly in the former state.

We divide the whole oversaturation period into two parts, the queuing period and the dissipation period. The queuing period is defined as the period when queue grows on at least one road, i.e.

$$
d_{1} / s_{1}+d_{2} / s_{2}>1 \text { or } d_{1} / s_{1}>g^{\max } / c \text { or } d_{2} / s_{2}>1-g^{\min } / c
$$

And the corresponding definition for the dissipation period is

$$
\left(d_{1}+n_{1} / c\right) / s_{1}+\left(d_{2}+n_{2} / c\right) / s_{2}>1 \text { and } d_{1} / s_{1}+d_{2} / s_{2} \leq 1 \text { and } d_{1} / s_{1} \leq g^{\max } / c \text { and } d_{2} / s_{2} \leq 1-g^{\min } / c
$$

Fig. 5 shows an example how the oversaturation period is divided. For simplicity, we assume in this example that if $d_{1} / s_{1}+d_{2} / s_{2} \leq 1, d_{1} / s_{1}>g^{\max } / c$ or $d_{2} / s_{2}>1-g^{\min } / c$ will not happen. Consequently, the oversaturation period, the queuing period and the dissipation period are identified by the value of $d_{1} / s_{1}+d_{2} / s_{2}$. At the time $t_{0}$, when the demand curve exceeds the maximum service line $d_{1} / s_{1}+d_{2} / s_{2} \equiv 1$, queue will be formed on one road or both. Till the time $t_{\mathrm{f}}$, the queues in both approaches are dissolved. So the oversaturation period is identified by the region $\left[t_{0}, t_{\mathrm{f}}\right]$. Furthermore, there is a critical time, $t_{\mathrm{c}}$, when the demand curve drops below the maximum service line. During $\left[t_{0}\right.$, $\left.t_{\mathrm{c}}\right]$, queues grow continuously, indicating the queuing period; during $\left[t_{\mathrm{c}}, t_{\mathrm{f}}\right]$, queues are possible to be dissolved gradually, indicating the dissipation period. 


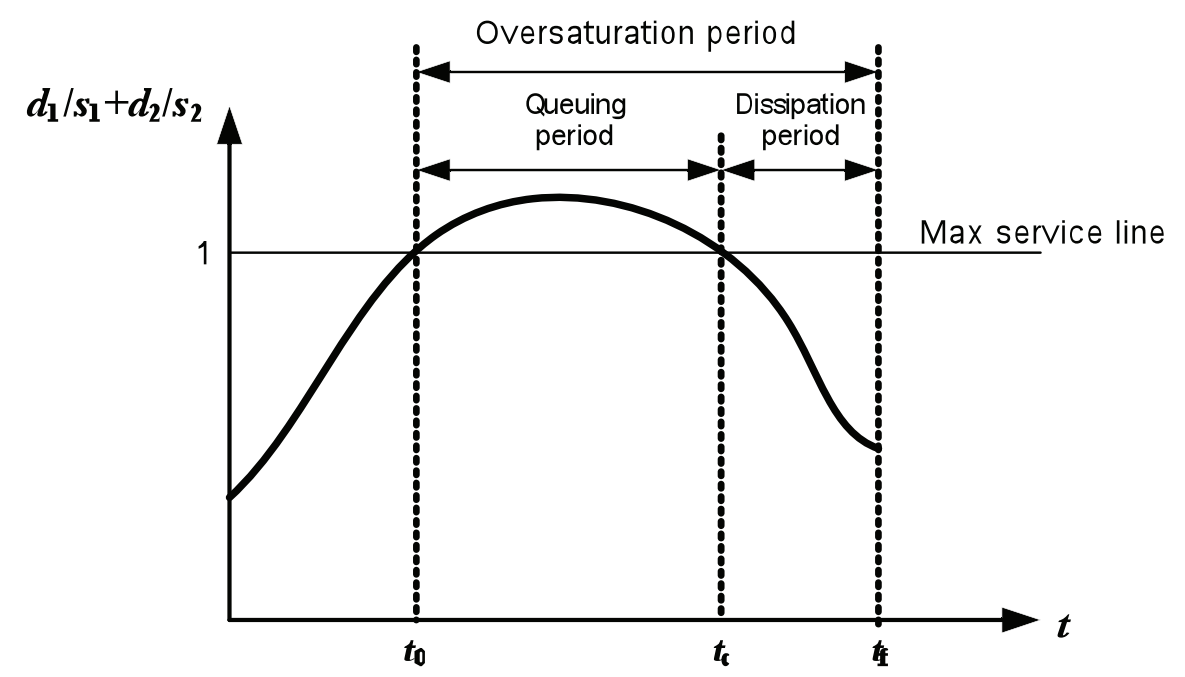

Fig. 5. Division of the oversaturation period: queuing period and dissipation period

Note that Fig. 5 only shows a simple example of how the oversaturation period is divided into the queueing and dissipation periods. More than one repetition of queuing and dissipation periods may take place. One will see in the following that such repetition will not impede the application of the QUEUE strategy.

The control objectives for these two periods are different. In the queuing period, the principal control objective is to prevent spillover, i.e., queue sizes should be restricted under the maximum value. But in the dissipation period, the principal control objective is to eliminate residual queues as soon as possible. Note again that we should also try to minimize the total delay as the secondary objective in the whole oversaturation period. In this paper, the control strategy for the dissipation period is discussed in detail, followed by the extension to the queuing period.

\subsection{QUEUE strategy for the dissipation period}

The idea of QUEUE strategy for the dissipation period comes intuitively from the queue state diagram (Fig. 3). Draw another two lines starting from the origin which are parallel to the boundaries starting from $\left[n_{1}{ }^{0}, n_{2}^{0}\right]$, respectively. The space between these two new boundaries indicates the attractable region of the origin. Then, the feasible region for trajectories that eliminate residual queues simultaneously at the earliest time is indicated by a parallelogram, as shown in Fig. 6.

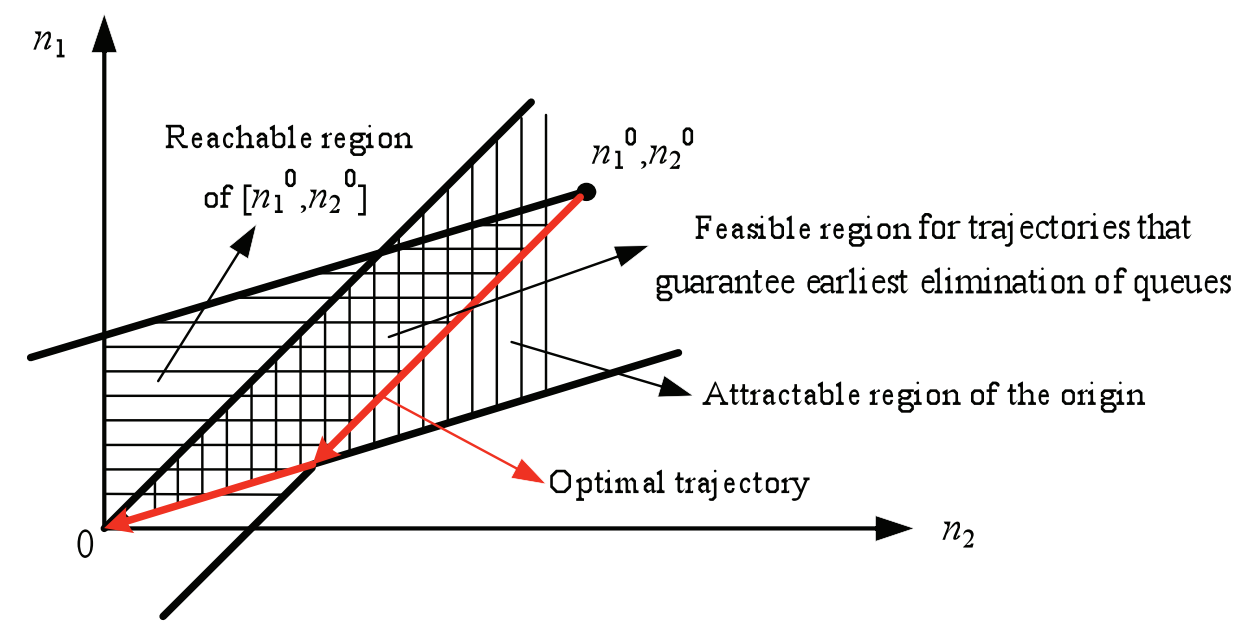

Fig. 6. The optimal trajectory during the dissipation period in case of constant demand

Since $s_{1}>s_{2}$, queues on road 1 should be served with high priority, the corresponding two-stage optimal trajectory of $\left[n_{1}, n_{2}\right]$ is depicted as red arrows in Fig. 6. This optimal trajectory shows clearly the logic of QUEUE, 
i.e., keep the maximum ratio of $\Delta n_{1} / \Delta n_{2}$ (let $g_{1}=g^{\text {max }}$ in other words), until the queue state $\left[n_{1}, n_{2}\right]$ hits the boundary line $n_{1} / n_{2}=\left(\Delta n_{1} / \Delta n_{2}\right)^{\mathrm{min}}$. The strategy is written as

$$
g_{1}= \begin{cases}g^{\max }, & \text { if } \frac{n_{1}}{n_{2}}>\left(\frac{\Delta n_{1}}{\Delta n_{2}}\right)^{\min } \\ g^{\min }, & \text { if } \frac{n_{1}}{n_{2}} \leq\left(\frac{\Delta n_{1}}{\Delta n_{2}}\right)^{\min }\end{cases}
$$

However, the preceding equation is not a complete strategy yet. Two exceptions exist.

The first one happens when the queues on the minor approach are possible to grow at the beginning of the dissipation period, i.e., $s_{2}\left(1-g^{\max } / \mathrm{c}\right)<B_{2}$, because the minor approach is assigned with minimum green time. In this condition, queues on the minor approach may exceed the maximum queue size with the current strategy given by (12). This reveals an intermediate transition period in which both the prevention of spillover and the elimination of residual queues should be considered. Under this condition, the queue size on the minor approach is examined first. If $n_{2}$ is far away from the maximum value, no additional treatment is needed; however if $n_{2}$ is in a vicinity of the maximum value, we must prevent $n_{2}$ from increasing any more. This amelioration is realized by increasing the minimum green time of the minor approach, or decreasing the maximum green time of the major approach in other words, to meet the current demand. The amelioration is written as

$$
g_{1}=c-B_{2} c / s_{2}, \text { if } n_{2}>n_{2}^{\text {threshold }}
$$

where $n_{2}{ }^{\text {threshold }}$ is the threshold value of queue size for the minor approach. It is easy to find that the value of $n_{2}$ would no longer increase, so queue spillover is prevented.

The second special condition that may change the form of two-stage bang-bang control has been mentioned by Gazis (1964). This condition happens when $s_{1} g^{\mathrm{min}} / \mathrm{c}<B_{1}$, and the two-stage CSC intersects the CDC on the major approach before the earliest end of the oversaturation period. Note that it will never happen on the minor approach, because $s_{2}\left(1-g^{\mathrm{min}} / \mathrm{c}\right) \geq B_{2}$ is given by the definition of the dissipation period. As shown in Fig. 7, the CSC joins the CDC on road 1 at time $\tau^{\prime}$ before the initially calculated switch time $\tau$. Because negative queue size is impossible, green time for road 1 is partially wasted during the time period $\left[\tau^{\prime}, \tau\right]$, and the queue size $n_{1}$ will increase but never goes to zero in the second stage $(\tau, T]$. So the optimal solution is modified under this condition. Graphically, the initially calculated CSC is clipped by the CDC on road 1 to give the optimal one. The corresponding feedback control strategy under this condition is

$$
g_{1}= \begin{cases}g^{\max }, & \text { if } n_{1}>0 \\ B_{1} c / s_{1}, & \text { if } n_{1}=0\end{cases}
$$




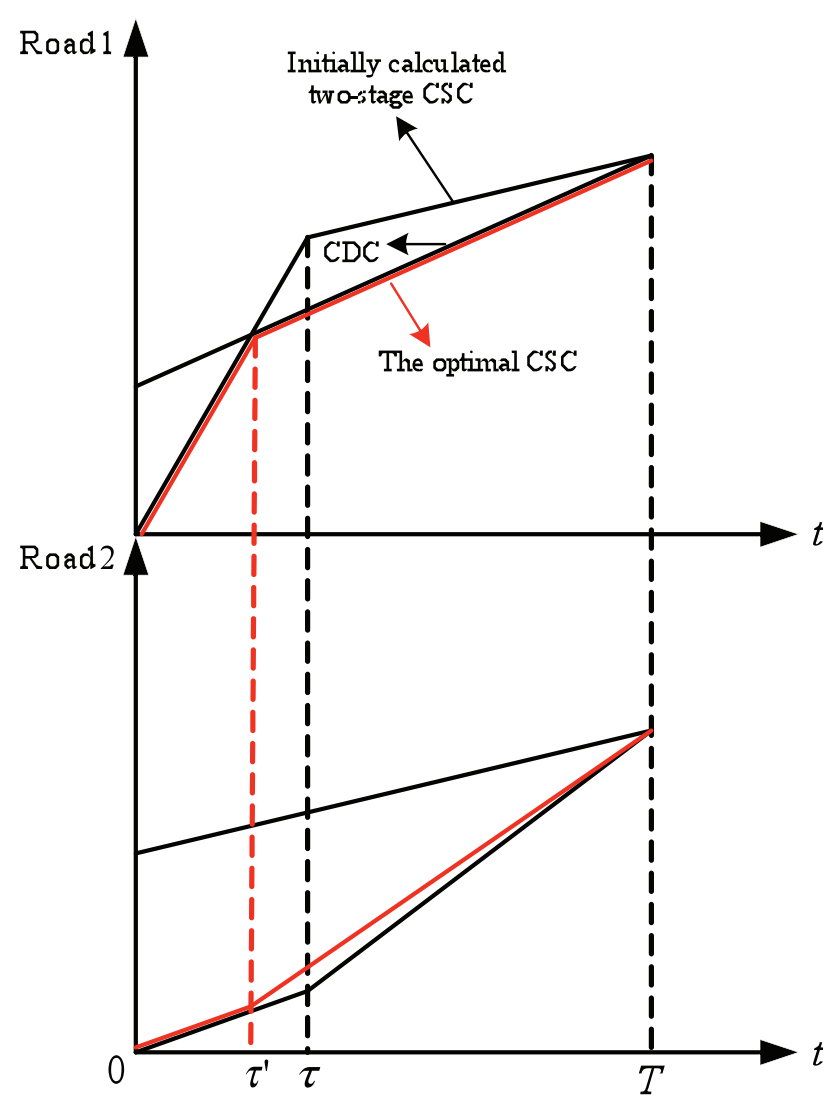

Fig. 7. The clipped two-stage optimal trajectories when $s_{1} g^{\mathrm{min}} / \mathrm{c}<B_{1}$

In conclusion, put (12)-(14) together, then the QUEUE strategy for the dissipation period reads

$$
g_{1}=\left\{\begin{array}{cc}
g^{\max }, \quad \text { if } s_{1} g^{\min } / c \geq B_{1} \text { and } \frac{n_{1}}{n_{2}}>\left(\frac{\Delta n_{1}}{\Delta n_{2}}\right)^{\text {min }} \text { and } n_{2} \leq n_{2}^{\text {threshold }}, \\
\quad \text { or } s_{1} g^{\min } / c<B_{1} \text { and } n_{1}>0 \text { and } n_{2} \leq n_{2}^{\text {threshold }} \\
-B_{2} c / s_{2}, \quad \text { if } s_{1} g^{\min } / c \geq B_{1} \text { and } \frac{n_{1}}{n_{2}}>\left(\frac{\Delta n_{1}}{\Delta n_{2}}\right)^{\text {min }} \text { and } n_{2}>n_{2}^{\text {threshold }}, \\
\text { or } s_{1} g^{\min } / c<B_{1} \text { and } n_{1}>0 \text { and } n_{2}>n_{2}^{\text {threshold }} \\
B_{1} c / s_{1}, \quad \text { if } s_{1} g^{\min } / c<B_{1} \text { and } n_{1}=0 \\
g^{\min ,} & \text { if } s_{1} g^{\min } / c \geq B_{1} \text { and } \frac{n_{1}}{n_{2}} \leq\left(\frac{\Delta n_{1}}{\Delta n_{2}}\right)^{\min }
\end{array}\right.
$$

The QUEUE strategy is simple, intuitive, and easy to be implemented cycle-by-cycle in real-world intersections. The only parameters needed are the current queue sizes and the arrival demands on both roads in the past cycle, which are readily observable with the help of various types of detectors. More importantly, the QUEUE strategy is a quasi-optimal strategy with respect to the ideal optimal one derived from off-line studies. The proposed QUEUE strategy for the dissipation period in case of constant arrival demand is proved to be optimal (see Appendix A for details), and the upper bounds of the sub-optimality are given in Section 4. 


\subsection{QUEUE strategy for the queuing period}

The QUEUE strategy for the queuing period has the similar logic with that for the dissipation period. Fig. 8 shows the optimal trajectory during the queuing period in the queue state plane. In comparison with Fig. 6, this trajectory goes in the opposite direction. The primary goal of the control strategy is to keep queue sizes within the maximum value $n_{1}{ }^{\max }$ and $n_{2}{ }^{\max }$ so that queues will not spill over to upstream intersections. Therefore, if in the extreme case that spillover has to happen, it should happen simultaneously on both roads at the latest time. The ideal optimal strategy in consideration with total delay time is also a two-stage bang-bang control strategy, in which the major road is given priority in the first stage. Similar to (12), the basic QUEUE strategy for the queuing period is written as

$$
g_{1}= \begin{cases}g^{\max }, & \text { if } \frac{n_{1}^{\max }-n_{1}}{n_{2}^{\max }-n_{2}}<\left(\frac{\Delta n_{1}}{\Delta n_{2}}\right)^{\min } \\ g^{\min }, & \text { if } \frac{n_{1}^{\max }-n_{1}}{n_{2}^{\max }-n_{2}} \geq\left(\frac{\Delta n_{1}}{\Delta n_{2}}\right)^{\min }\end{cases}
$$

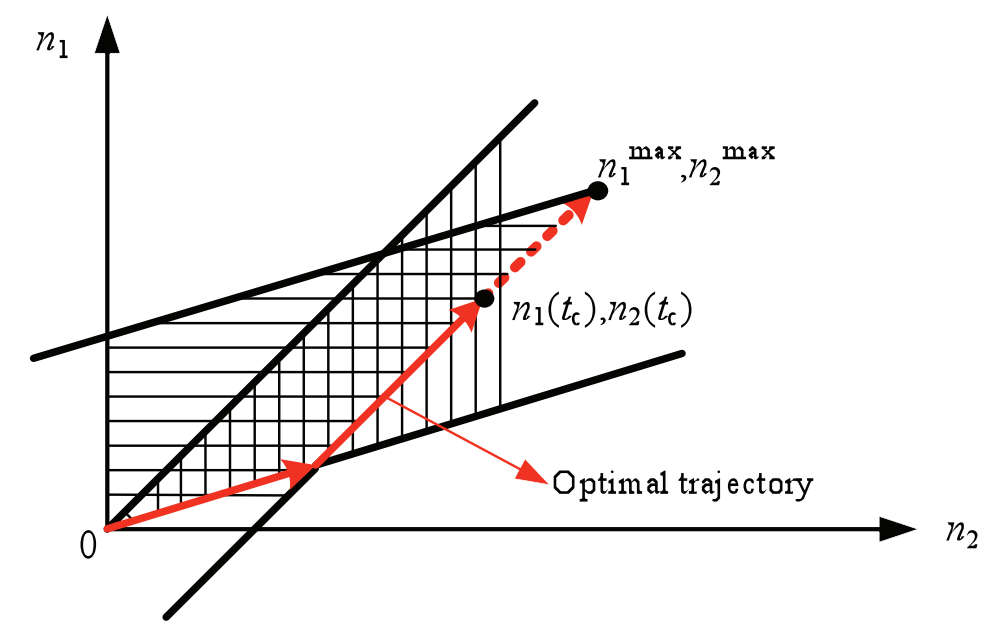

Fig. 8. The optimal trajectory during the queuing period in case of constant demand

Note that it is not necessary for the trajectory to reach $\left[n_{1}{ }^{\max }, n_{2}{ }^{\max }\right]$. In most cases, the trajectory ends at $\left[n_{1}\left(t_{\mathrm{c}}\right), n_{2}\left(t_{\mathrm{c}}\right)\right]$, where $t_{\mathrm{c}}$ is the time when the queuing period ends. However, the destination of the trajectory should be set to $\left[n_{1}{ }^{\max }, n_{2}{ }^{\max }\right]$ in all cases so that the maximum queue size constraints are satisfied with most possibility.

In comparison with the earliest end time of queue dissipation, the latest start time of queue spillover is much more essential. A further profitable trade-off can be made regarding the maximum queue constraints. The idea is to reduce the possibility of spillover by increasing some total delay time. It is realized by making the queue size constraints stricter, so that some queuing spaces, denoted $n_{1}{ }^{\text {res }}$ and $n_{2}{ }^{\text {res }}$, are reserved to deal with unpredictable demand variations. Then the queue size constraints are compressed to $\left[n_{1}{ }^{\max }-n_{1}{ }^{\text {res }}, n_{2}{ }^{\text {max }}-n_{2}{ }^{\text {res }}\right]$. Even if the compressed constraints are broken, it is still possible to satisfy the maximum queue size constraints $\left[n_{1}{ }^{\max }, n_{2}{ }^{\max }\right]$ because of the maneuverability within the reserved queuing spaces. Fig. 9 gives an example. Fig. 9(a) shows the case without reserved queuing spaces. It is clear that the queue state point has already gone out of the final boundaries (red line) of the feasible region of $\left[n_{1}{ }^{\max }, n_{2}{ }^{\max }\right]$ when demand changes, so the maximum queue size constraint is broken. The comparative result with reserved queuing spaces is shown in Fig. 9(b). The compressed constraint makes the control trajectory switch to the second stage earlier. As a result, the queue size state still lies within the final boundaries of the feasible region of $\left[n_{1}{ }^{\max }, n_{2}{ }^{\mathrm{max}}\right]$ when the demand changes. Although the 
compressed constraint is broken, the controlled trajectory is still capable to reach the destination $\left[n_{1}{ }^{\max }, n_{2}{ }^{\max }\right]$ without breaking the maximum queue constraint.
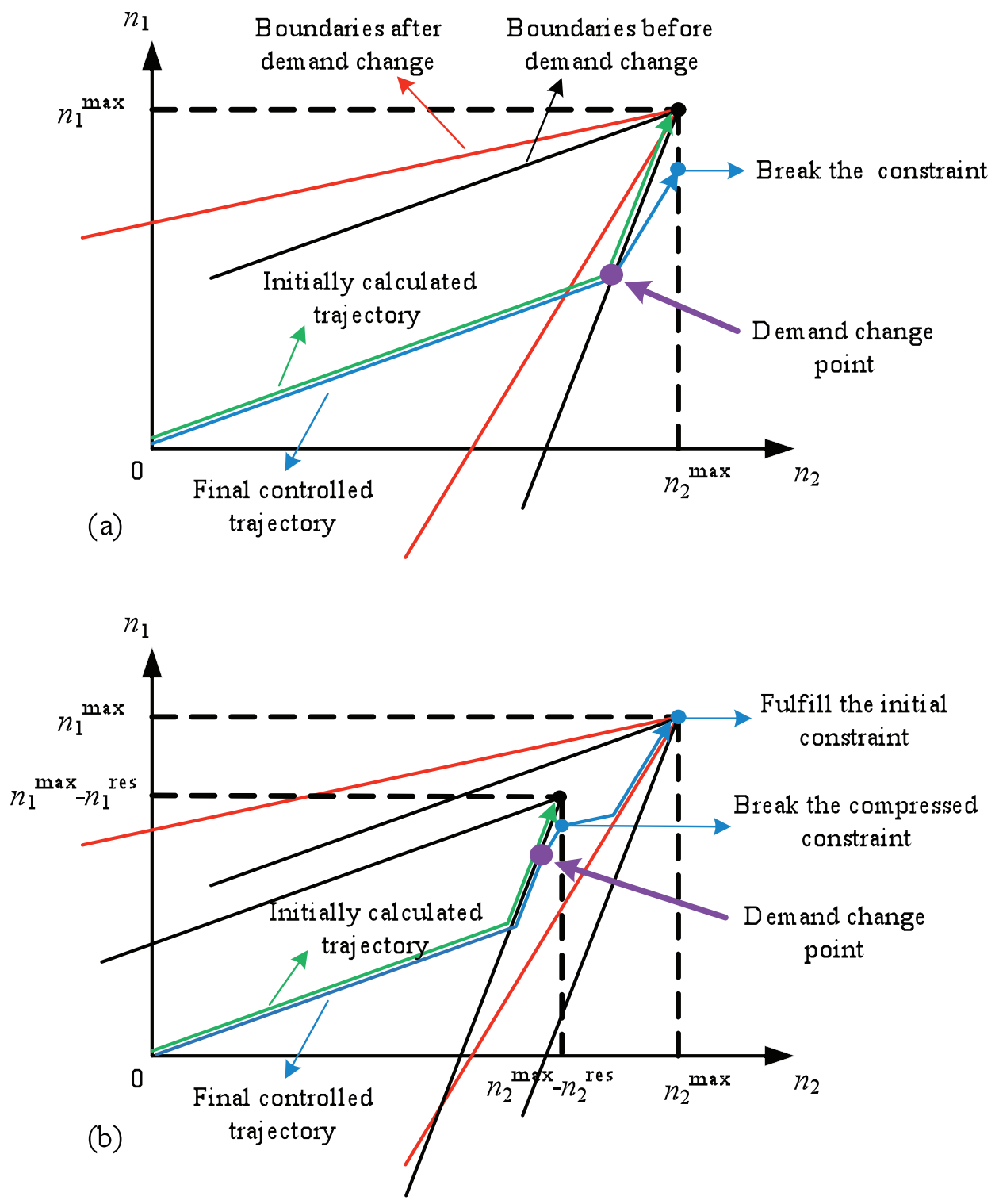

Fig. 9. Comparison of the QUEUE strategy (a) the initial one; (b) with reserved queuing spaces

Note that the reserved queuing space plays the same role as the aforementioned threshold queue size does. Both of them are adopted to prevent spillover caused by random fluctuation of the demand. For simplicity, we can let $n_{1}{ }^{\text {threshold }}=n_{1}{ }^{\text {max }}{ }_{-} n_{1}{ }^{\text {res }}$ and $n_{2}{ }^{\text {threshold }}=n_{2}{ }^{\text {max }}-n_{2}$ res , then $n_{1}{ }^{\text {res }}$ and $n_{2}{ }^{\text {res }}$ are the only set values needed by the signal controller. The value of $n_{1}$ res and $n_{2}{ }^{\text {res }}$ should be selected according to the historical knowledge from the real world. Their value should be set larger if the demand tends to change with larger amplitude, or if the intersection has a longer signal cycle.

\subsection{Summary of the QUEUE strategy}

The cycle-by-cycle procedure of the QUEUE strategy for the whole oversaturation period is summarized as follows:

Step 0) Determine the values of reserved queuing space, $n_{1}{ }^{\text {res }}$ and $n_{2}{ }^{\text {res }}$.

Step 1) At the beginning of each cycle, detect the current queue sizes $n_{1}$ and $n_{2}$, and the arrival demand $d_{1}$ and $d_{2}$ in the past cycle. Check according to (2) (10) and (11) which period the current state belongs to. 
Step 2) Determine the green split for the coming cycle. Go to Step 1 when next cycle starts.

\section{Bounding the sub-optimality of the QUEUE strategy}

The performance of the QUEUE strategy is assessed quantitatively in this section by finding the upper bounds of sub-optimality in comparison with the off-line optimum. Unlike the previous section, the assumption of constant demand is relaxed to piecewise constant which is acceptable to describe the fluctuating real world demand. With the demand changing unpredictably, it is impossible for any on-line control strategy to perfectly match the ideal off-line optimum. Fortunately, the result derived by the QUEUE strategy is found much close to the optimal one. Note that only the dissipation period is considered here. The same procedure can be applied to the queuing period, but is omitted in this paper.

The sub-optimality arises from the unpredictable demand change. The change of demands will influence the slopes of boundaries (see Fig. 3) initially calculated by (9). When the demands are time varying, the slopes are

$$
\begin{aligned}
& \left(\frac{\Delta n_{1}}{\Delta n_{2}}\right)^{\min }=\frac{d_{1}-s_{1} g^{\min } / c}{d_{2}-s_{2}\left(1-g^{\min } / c\right)} \\
& \left(\frac{\Delta n_{1}}{\Delta n_{2}}\right)^{\max }=\frac{d_{1}-s_{1} g^{\max } / c}{d_{2}-s_{2}\left(1-g^{\max } / c\right)}
\end{aligned}
$$

The slopes may increase or decrease as the demands $d_{1}$ and $d_{2}$ fluctuate. In the dissipation period, it is reasonable to assume that the demands only change in descending direction. Consequently, the demand changes are categorized into three types: Type 1 for $d_{1}$ remains constant but $d_{2}$ decreases, Type 2 for $d_{2}$ remains constant but $d_{1}$ decreases, and Type 3 for both $d_{1}$ and $d_{2}$ decrease. Overall, the demand changes consist of a series of change Type 1, Type 2 or Type 3 . To bound the sub-optimality of the QUEUE strategy, we only need to consider Type 1 and Type 2 with the following two extreme cases:

1) $d_{1}$ remains constant $\left(d_{1} \equiv B_{1}\right)$ but $d_{2}$ changes directly to zero $\left(d_{2}=B_{2} \rightarrow d_{2}=0\right)$;

2) $d_{2}$ remains constant $\left(d_{2} \equiv B_{2}\right)$ but $d_{1}$ changes directly to zero $\left(d_{1}=B_{1} \rightarrow d_{1}=0\right)$.

\subsection{Upper bound Type 1: $d_{1}$ remains constant but $d_{2}$ changes directly to zero}

First, we assume that $s_{1} g^{\mathrm{min}} / \mathrm{c} \geq B_{1}$. The case is depicted in Fig. 10. The initially estimated CDCs and CSCs calculated by the QUEUE strategy are depicted with black lines. The calculated value of end time and switch time are $T^{\prime}$ and $\tau^{\prime}$ that given by (7) and (8), respectively. Now we assume that $d_{2}$ changes suddenly to zero at time $t_{\mathrm{s}}$. The actual CDCs and the off-line optimal trajectories of CSCs are depicted with red lines. The off-line optimal value of $T^{*}$ and $\tau^{*}$ are marked. It is easy to know that the decrease of $d_{2}$ always causes an earlier end of the oversaturation period and a later switch time. 


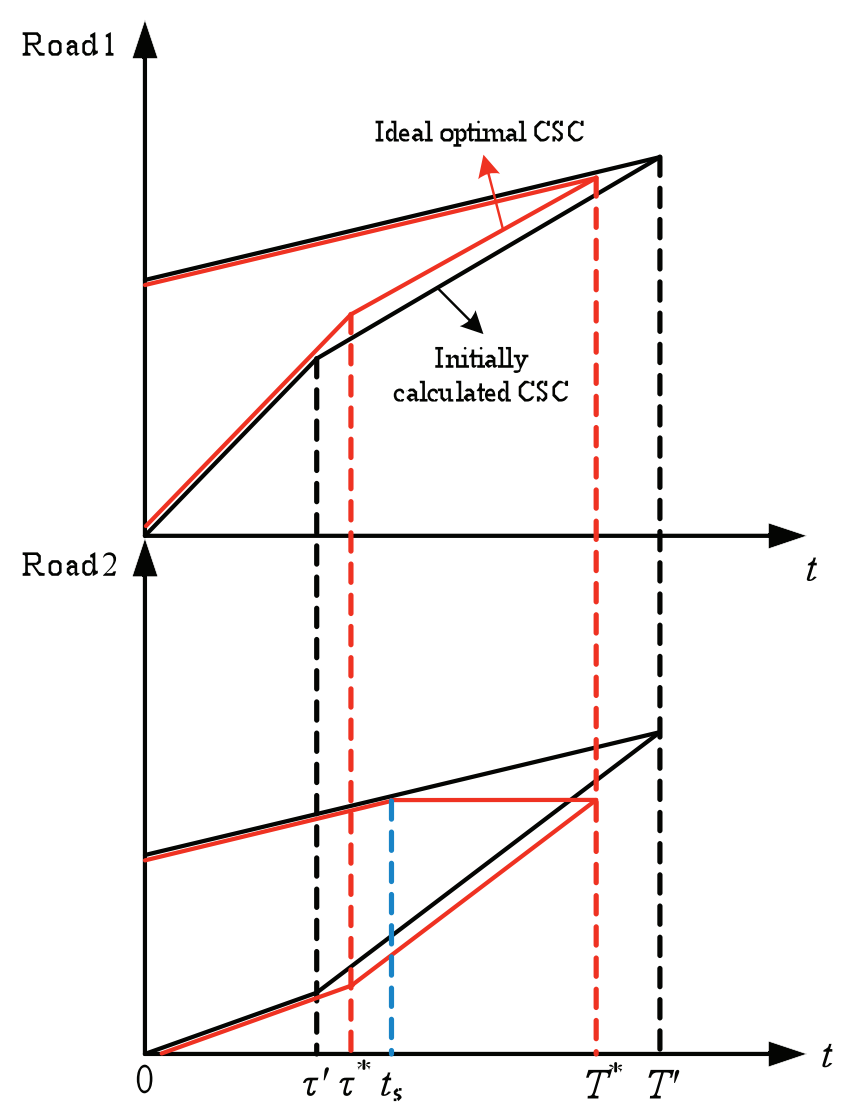

Fig. 10. The case when $d_{1}$ remains constant but $d_{2}$ changes suddenly to zero

Unaware of the demand change in advance, the actual trajectories of CSCs follow the initially calculated black curves before $t_{\mathrm{s}}$. Consequently, if $t_{\mathrm{s}} \leq \tau$ ', the proposed algorithm will not deviate from the ideal optimum and is able to postpone the switch time $\tau^{\prime}$ till the optimal time $\tau^{*}$. So the QUEUE strategy can still generate the optimal solution. But if $t_{\mathrm{s}}>\tau^{\prime}$, i.e., the switch has already been made at time $\tau^{\prime}$, it is impossible for the algorithm to go back and extend the first stage. Under this circumstance, the proposed algorithm restarts the first stage at $t_{\mathrm{s}}$, and switch back to the second stage afterwards. A distinct comparison of the ideal optimum, the initial solution and the final solution derived by the algorithm is depicted in Fig. 11. For the sake of a clear view, overlapped lines are drawn separately with small gaps. The red curve shows the ideal optimal one, which is different with the initial solution (the black curve) in both end time and switch time. Before $t_{\mathrm{s}}$, the final solution (the blue curve) is calculated based on the initial demand, and follows the trajectory of the initial one. But at $t_{\mathrm{s}}$ (note again $t_{\mathrm{s}}>\tau^{\prime}$ ) when the changed demand is detected, the value of $\left(\Delta n_{1} / \Delta n_{2}\right)^{\text {min }}$ decreases and drops below the value of $n_{1} / n_{2}$, so the first stage is restarted, until a further switch time $\tau^{\prime \prime}$. It is easy to find that $\tau^{\prime \prime}-t_{\mathrm{s}}=\tau^{*}-\tau^{\prime}$. Consequently, the final solution joins the off-line optimal trajectory at time $\tau$ "' and then the two curves follow the same way to the earliest end of oversaturation. 


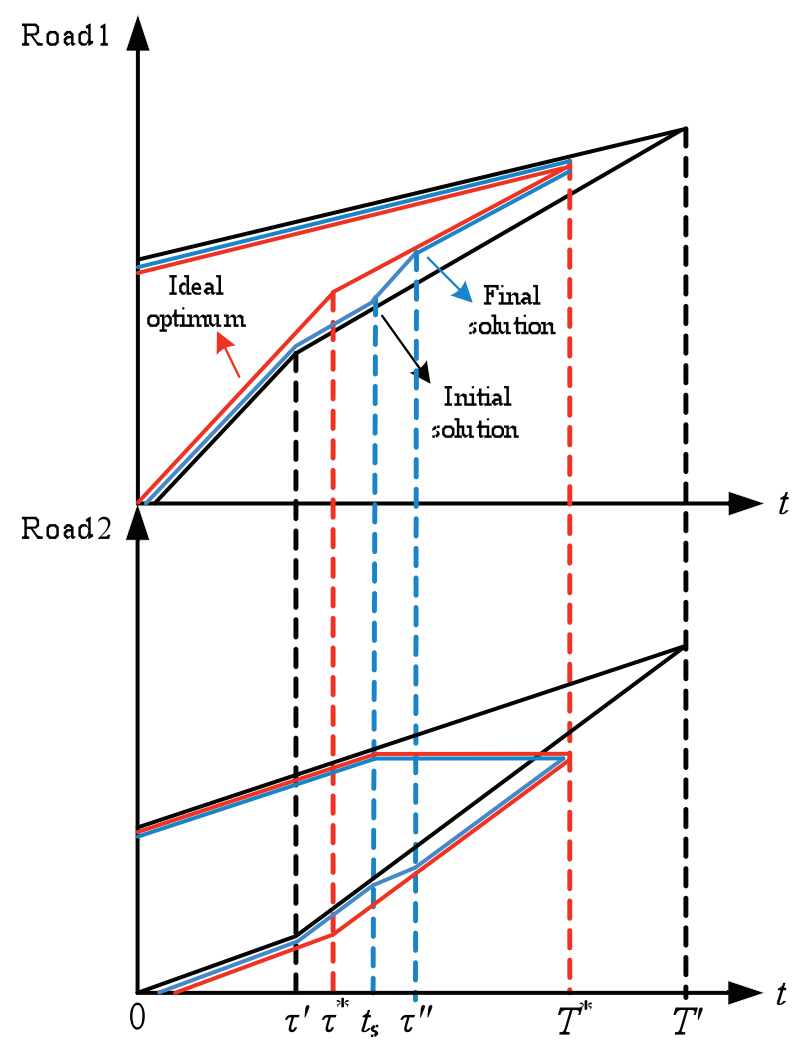

Fig. 11. A distinct comparison of the ideal optimum, the initial and final solution of CSC on road 1

In this case, the final solution of the proposed algorithm can still catch the earliest end of oversaturation to fulfill the principal control objective. The only drawback comparing to the off-line optimum is some increase of total delay time, indicated by the area of parallelogram between the blue and red curves in Fig. 11. Geometrically, the difference of delay time on road $1, \Delta D T_{1}$ is calculated as follows:

$$
\Delta D T_{1}=s_{1} \frac{g^{\max }-g^{\min }}{c}\left(\tau^{*}-\tau^{\prime}\right)\left(t_{s}-\tau^{\prime}\right)
$$

Similarly, the difference of delay time on $\operatorname{road} 2, \Delta D T_{2}$, is calculated as follows:

$$
\Delta D T_{2}=s_{2} \frac{g^{\min }-g^{\max }}{c}\left(\tau^{*}-\tau^{\prime}\right)\left(t_{s}-\tau^{\prime}\right)
$$

So the difference of total delay time, $\triangle T D T$ is

$$
\Delta T D T=D T_{1}+D T_{2}=\left(s_{1}-s_{2}\right) \frac{g^{\max }-g^{\min }}{c}\left(\tau^{*}-\tau^{\prime}\right)\left(t_{s}-\tau^{\prime}\right)=M\left(t_{s}-\tau^{\prime}\right)\left(T^{\prime}-t_{s}\right)
$$

where, $M$ is constant, $M=\frac{\left(s_{1}-s_{2}\right)\left[s_{1} g^{\min }-c B_{1}\right] B_{2}}{c\left(s_{1} s_{2}-B_{1} s_{2}\right)}>0$.

(20) shows that $\triangle T D T$ is a parabola function of $t_{\mathrm{s}}$, within the domain $t_{\mathrm{s}} \in\left[\tau^{\prime}, T^{\prime}\right]$. Thus, it is easy to find that the maximum value of $\triangle T D T$, denoted by $\Delta T D T^{\max }$, is derived when $t_{\mathrm{s}}=\left(\tau^{\prime}+T^{\prime}\right) / 2$, and that

$$
\Delta T D T^{\max }=M \cdot \frac{\left(T^{\prime}-\tau^{\prime}\right)^{2}}{4}=\frac{\left(s_{1}-s_{2}\right)\left[s_{1} g^{\min }-c B_{1}\right] B_{2}\left(T^{\prime}-\tau^{\prime}\right)^{2}}{4 c\left(s_{1} s_{2}-B_{1} s_{2}\right)}
$$


On the other hand, if $s_{1} g^{\mathrm{min}} / \mathrm{c}<B_{1}$, no matter when the value of $d_{2}$ drops to zero, the initially calculated two-stage bang-bang strategy will be clipped by the CDC on road 1, which is unchanged. Therefore, the off-line optimal strategy remains the same, i.e., keeping $g_{1}=g^{\max }$ until the CSC joins CDC on approach 1 . So the feedback strategy (14) coincides with the off-line optimal strategy in this case.

Overall, the Type 1 change of demand will not delay the earliest end time of the oversaturation period, and the upper bound for increased total delay time with respect to the off-line optimum is described by (21).

\subsection{Upper bound Type 2: $d_{2}$ remains constant but $d_{1}$ changes directly to zero}

First, we still assume that $s_{1} g^{\mathrm{min}} / \mathrm{c} \geq B_{1}$. Note that this inequality still holds when $d_{1}$ decreases. The case is depicted in Fig. 12. Similarly, the initially CDCs and CSCs calculated by the QUEUE strategy, and the corresponding $T^{\prime}$ and $\tau^{\prime}$, are depicted with black lines. Now we assume that $d_{1}$ changes suddenly to zero at time $t_{\mathrm{s}}$, and $t_{\mathrm{s}} \geq \tau^{\prime}$. The actual CDCs, the ideal optimal trajectories of CSCs, and the ideal optimal value of $T^{*}$ and $\tau^{*}$ are depicted with red lines. In this case, the decrease of $d_{1}$ causes an earlier end of the oversaturation period and an earlier switch time.

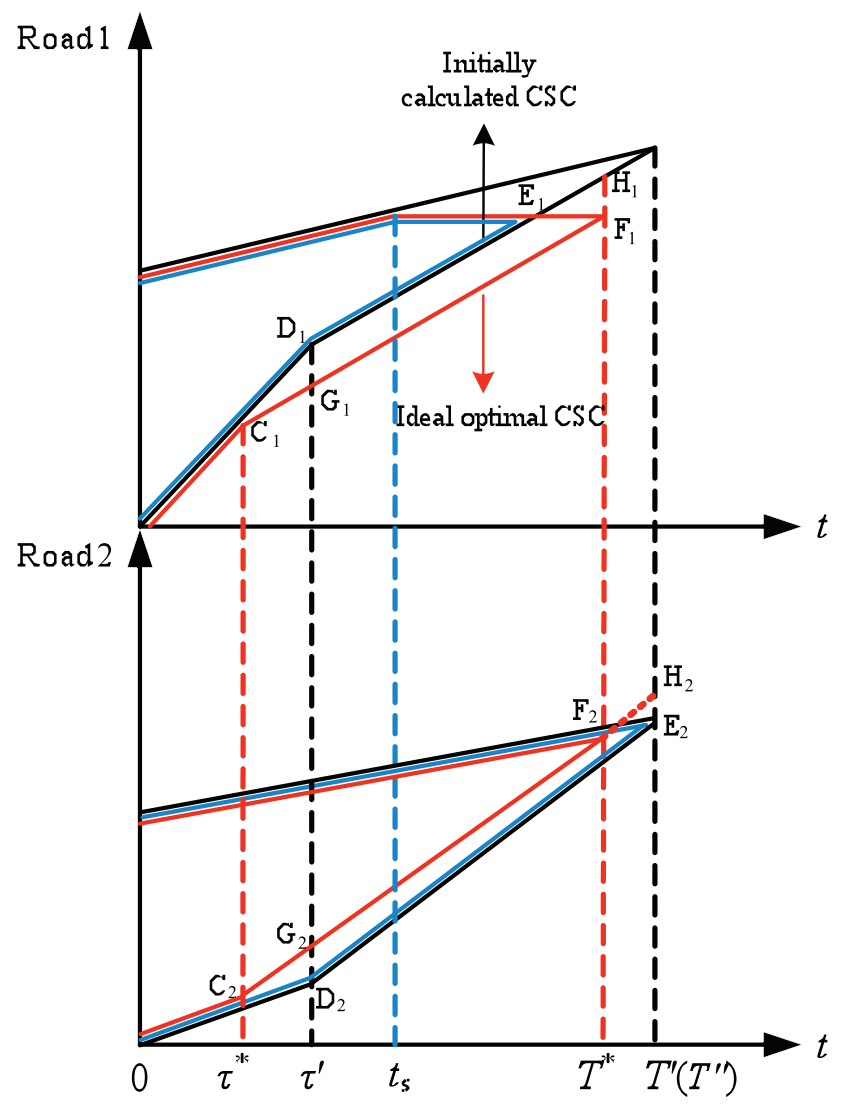

Fig. 12. The case when $d_{2}$ remains constant but $d_{1}$ changes suddenly to zero: $t_{\mathrm{s}} \geq \tau$,

The sudden drop of $d_{1}$ indicates that stage 2 should start earlier, or shorten the stage 1 in other words, which is unfortunately unreachable. On-line feedback control algorithms can do nothing but to maintain the initial solution. Consequently, the queues do not disappear simultaneously on both routes. The time when all queues are dissolved, denoted by $T^{\prime}$ ', equals to $T^{\prime}$ 'in this case, and is later than the ideal earliest one $T^{*}$. Note here the value of $T$ ' is given by (7), and the value of $T^{*}$ can be calculated similarly as follows:

$$
T^{*}=\frac{A_{1} s_{2}+A_{2} s_{1}}{s_{1} s_{2}-B_{1}^{\prime} s_{2}-B_{2} s_{1}}=\frac{A_{1} s_{2}+A_{2} s_{1}}{s_{1} s_{2}-B_{2} s_{1}}+\frac{B_{1} s_{2}}{s_{1} s_{2}-B_{2} s_{1}} \cdot t_{s}
$$


where $B_{1}$ ' is the equivalent constant demand rate with which the optimal two-stage solution is the same as that shown in Fig. 12. $B_{1}$ ' satisfies the following equation.

$$
A_{1}+B_{1}^{\prime} T^{*}=A_{1}+B_{1} t_{s}
$$

Then, the delayed ending time, $\Delta T$, is calculated as follows:

$$
\Delta T=T^{\prime \prime}-T^{*}=\frac{A_{1} s_{2}+A_{2} s_{1}}{s_{1} s_{2}-B_{1} s_{2}-B_{2} s_{1}}-\left(\frac{A_{1} s_{2}+A_{2} s_{1}}{s_{1} s_{2}-B_{2} s_{1}}+\frac{B_{1} s_{2}}{s_{1} s_{2}-B_{2} s_{1}} \cdot t_{s}\right)=\frac{B_{1} s_{2}}{s_{1} s_{2}-B_{2} s_{1}}\left(T^{\prime}-t_{s}\right)
$$

It is easy to see that $\Delta T$ is a linear descending function of $t_{\mathrm{s}}$. With the assumption that $t_{\mathrm{s}} \geq \tau^{\prime}$, the maximum value of $\Delta T$, denoted by $\Delta T^{\max }$, is derived when $t_{\mathrm{s}}=\tau^{\prime}$, and that

$$
\Delta T^{\max }=\frac{B_{1} s_{2}}{s_{1} s_{2}-B_{2} s_{1}}\left(T^{\prime}-\tau^{\prime}\right)
$$

On the other hand when $t_{\mathrm{s}}<\tau$, two possibilities exist. If $t_{\mathrm{s}} \leq \tau^{*}$, the QUEUE strategy is optimal as discussed before. However, if $t_{\mathrm{s}}=t_{\mathrm{a}} \in\left(\tau^{*}, \tau^{\prime}\right)$, we should calculate the delayed end time and see whether (25) is still the maximum value. In this case, the QUEUE strategy has not switched to the second stage when the demand change is detected at $t_{\mathrm{s}}$. As the updated value of $\left(\Delta n_{1} / \Delta n_{2}\right)^{\min }$ is less than the value of $n_{1} / n_{2}$, the second stage is started immediately. As shown in Fig. 13, the final trajectory derived from the QUEUE strategy lies between the initially calculated one and the ideal optimal one.

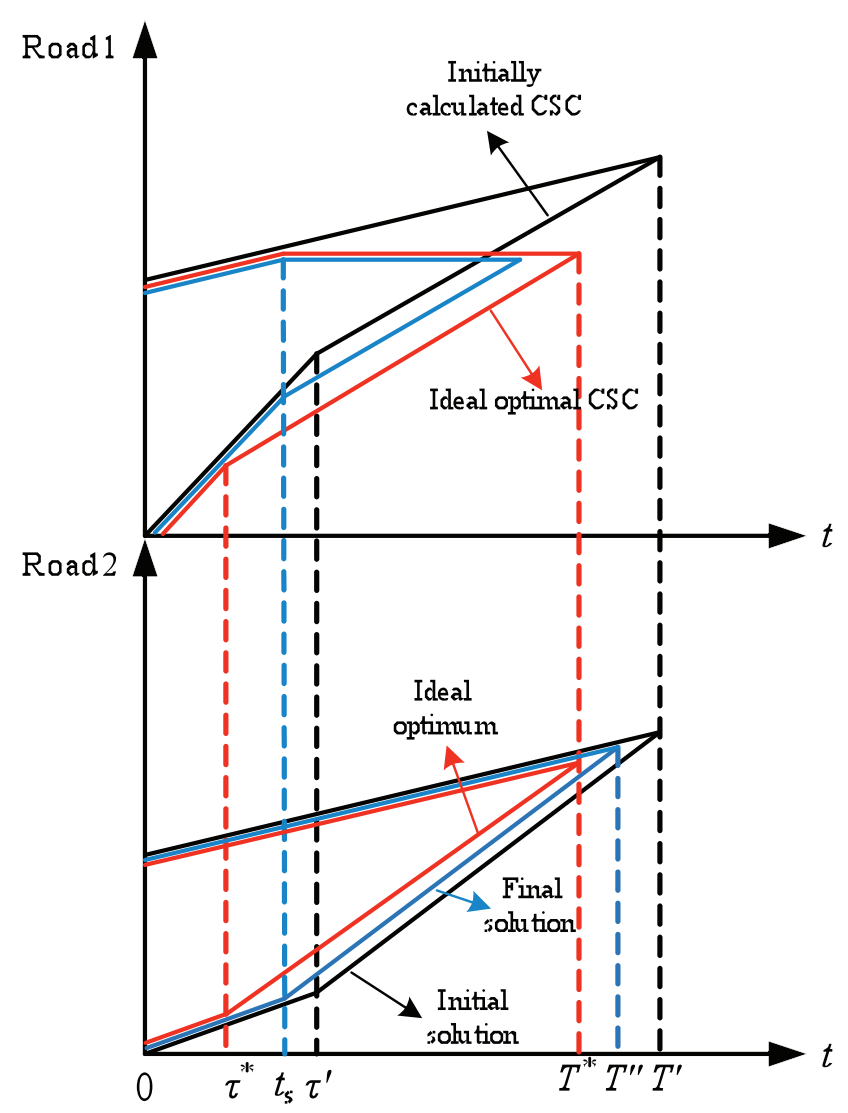

Fig. 13. The case when $d_{2}$ remains constant but $d_{1}$ changes suddenly to zero: $t_{\mathrm{s}}<\tau$ '

Now we are going to compare the delayed ending time $\Delta T$ in this case with the maximum value given by (25). Note that the value of $\Delta T$ is a function of $t_{\mathrm{s}}$, and the maximum value given by (25) is derived when $t_{\mathrm{s}}=\tau^{\prime}$. So, the 
difference between these two values is

$$
\Delta T\left(t_{\mathrm{a}}\right)-T\left(\tau^{\prime}\right)=\left(T^{\prime \prime}\left(t_{\mathrm{a}}\right)-T^{*}\left(t_{\mathrm{a}}\right)\right)-\left(T^{\prime \prime}\left(\tau^{\prime}\right)-T^{*}\left(\tau^{\prime}\right)\right)=\left(T^{\prime \prime}\left(t_{\mathrm{a}}\right)-T^{\prime \prime}\left(\tau^{\prime}\right)\right)-\left(T^{*}\left(t_{\mathrm{a}}\right)-T^{*}\left(\tau^{\prime}\right)\right)
$$

The value of $T^{\prime \prime}\left(t_{\mathrm{a}}\right)-T^{\prime \prime}\left(\tau^{\prime}\right)$ is calculated using graphical method:

$$
T^{\prime \prime}\left(t_{\mathrm{a}}\right)-T^{\prime \prime}\left(\tau^{\prime}\right)=\left(t_{\mathrm{a}}-\tau^{\prime}\right) \cdot \frac{s_{2}\left(1-g^{\min } / c\right)-B_{2}}{s_{2}\left(1-g^{\min } / c\right)-s_{2}\left(1-g^{\max } / c\right)}
$$

Note again that $s_{2}\left(1-g^{\max } / \mathrm{c}\right) \geq B_{2}$ is assumed, and $t_{\mathrm{a}}-\tau^{\prime}<0$, so we have

$$
T^{\prime \prime}\left(t_{\mathrm{a}}\right)-T^{\prime \prime}\left(\tau^{\prime}\right) \leq\left(t_{\mathrm{a}}-\tau^{\prime}\right) \cdot \frac{s_{2}\left(1-g^{\min } / c\right)-s_{2}\left(1-g^{\max } / c\right)}{s_{2}\left(1-g^{\min } / c\right)-s_{2}\left(1-g^{\max } / c\right)}=t_{\mathrm{a}}-\tau^{\prime}
$$

The value of $T^{*}\left(t_{\mathrm{a}}\right)-T^{*}\left(\tau^{\prime}\right)$ is calculated based on (22):

$$
T^{*}\left(t_{\mathrm{a}}\right)-T^{*}\left(\tau^{\prime}\right)=\left(t_{\mathrm{a}}-\tau^{\prime}\right) \cdot \frac{B_{1} s_{2}}{s_{1} s_{2}-B_{2} s_{1}}
$$

Note that $s_{1} g^{\mathrm{min}} / \mathrm{c} \geq B_{1}$ is assumed, so we have

$$
T^{*}\left(t_{\mathrm{a}}\right)-T^{*}\left(\tau^{\prime}\right) \geq\left(t_{\mathrm{a}}-\tau^{\prime}\right) \cdot \frac{s_{1} g^{\min } / c \cdot s_{2}}{s_{1} s_{2}-s_{2}\left(1-g^{\max } / c\right) \cdot s_{1}}=\left(t_{\mathrm{a}}-\tau^{\prime}\right) \cdot \frac{g^{\min }}{g^{\max }}>t_{\mathrm{a}}-\tau^{\prime}
$$

Consequently,

$$
\Delta T\left(t_{s}\right)-T\left(\tau^{\prime}\right)=\left(T^{\prime \prime}\left(t_{s}\right)-T^{\prime \prime}\left(\tau^{\prime}\right)\right)-\left(T^{*}\left(t_{s}\right)-T^{*}\left(\tau^{\prime}\right)\right)<0
$$

Therefore, (25) gives the maximum delayed ending time when $s_{1} g^{\mathrm{min}} / \mathrm{c} \geq B_{1}$, no matter when the demand change takes place.

For the case when $s_{1} \mathrm{~g}^{\mathrm{min}} / \mathrm{c}<B_{1}$, the expression of the delayed ending time $\Delta T$ is different. We are to compare the value of $\Delta T$ in this case with that calculated by (24). As $\Delta T=T^{\prime \prime}{ }^{\prime} T^{*}$, the ideal earliest end time $T^{*}$ and the actual end time $T^{\prime \prime}$ are compared separately. First, the expression of the earliest end time $T^{*}$ (see (22) ) shows that it is only influenced by parameters of the CDCs $\left(A_{i}, B_{i}\right.$ and $\left.t_{\mathrm{s}}\right)$, and the saturation service rate $s_{i}$. So the value of $T^{*}$ will not change whether the CSC is clipped by the CDC or not. Second, the actual end time $T$ "' is earlier in the case than that when $s_{1} g^{\mathrm{min}} / \mathrm{c} \geq B_{1}$. This is because that, no matter the QUEUE strategy coincides with the optimal solution or switches to the second stage immediately at $t_{\mathrm{s}}$, the clipped CSC on road 2 is always above or equals to the initial two-stage one before $t_{\mathrm{s}}$ (see Fig. 7). Consequently, the value of $\Delta T$ that calculated with the clipped CSC is always less than or equals to the calculated assuming ideal two-stage optimal CSC. So (25) gives the maximum value of $\Delta T$ no matter the initially calculated CSC is clipped or not.

Lastly, the value of total delay time when $\Delta T=\Delta T^{\max }$ is examined. This value is compared to that when the earliest end time is derived by the off-line optimal strategy. As shown in Fig. 12, the difference of total delay time $\triangle T D T$ is calculated by the difference between the area of trapezoid $\mathrm{C}_{1} \mathrm{D}_{1} \mathrm{E}_{1} \mathrm{~F}_{1}$ and $\mathrm{C}_{2} \mathrm{D}_{2} \mathrm{E}_{2} \mathrm{~F}_{2}$. For simplicity, we assume that the area of triangle $\mathrm{C}_{1} \mathrm{D}_{1} \mathrm{G}_{1}$ approximately equals that of triangle $\mathrm{E}_{1} \mathrm{~F}_{1} \mathrm{H}_{1}$, and the area of triangle $\mathrm{C}_{2} \mathrm{D}_{2} \mathrm{G}_{2}$ approximately equals that of triangle $\mathrm{E}_{2} \mathrm{~F}_{2} \mathrm{H}_{2}$. Thus,

$$
\begin{aligned}
\Delta T D T=D T_{1}+D T_{2} & \approx \frac{-\left(T^{*}-\tau^{\prime}\right) s_{1}\left(\tau^{\prime}-\tau^{*}\right)\left(g^{\max }-g^{\min }\right)}{c}+\frac{\left(T^{\prime}-\tau^{\prime}\right) s_{2}\left(\tau^{\prime}-\tau^{*}\right)\left(g^{\max }-g^{\min }\right)}{c} \\
& \left.=\frac{\left(\tau^{\prime}-\tau^{*}\right)\left(g^{\max }-g^{\min }\right)}{c} \cdot\left[T^{\prime}-\tau^{\prime}\right) s_{2}-\left(T^{*}-\tau^{\prime}\right) s_{1}\right]
\end{aligned}
$$


Note that $\tau^{\prime}>\tau^{*}$ and $g^{\max }>g^{\min }$, the sign of $\triangle T D T$ is determined by the sign of $\left(T^{\prime}-\tau^{\prime}\right) S_{2}-\left(T^{*}-\tau^{\prime}\right) s_{1}$, or the sign of $s_{2} / s_{1}-\left(T^{*}-\tau^{\prime}\right) /\left(T^{\prime}-\tau^{\prime}\right)$. Note also that $T^{*}$ is calculated when $t_{\mathrm{s}}=\tau^{\prime}$, so we have the following:

$$
\frac{T^{*}-\tau^{\prime}}{T^{\prime}-\tau^{\prime}}=1-\frac{T^{\prime}-T^{*}}{T^{\prime}-\tau^{\prime}}=1-\frac{\Delta T^{\max }}{T^{\prime}-\tau^{\prime}}=1-\frac{B_{1} s_{2}}{s_{1} s_{2}-B_{2} s_{1}} \geq 1-\frac{s_{1} g^{\min } / c \cdot s_{2}}{s_{1} s_{2}-s_{2}\left(1-g^{\max } / c\right) \cdot s_{1}}=1-\frac{g^{\min }}{g^{\max }}
$$

Generally, the value of $g^{\min } / g^{\max }$ is very small. If we assume $g^{\min } / g^{\max }<\left(s_{1}-s_{2}\right) / s_{1}$, (33) is further written as

$$
\frac{T^{*}-\tau^{\prime}}{T^{\prime}-\tau^{\prime}}>1-\frac{s_{1}-s_{2}}{s_{1}}=\frac{s_{2}}{s_{1}}
$$

Consequently, $s_{2} / s_{1}-\left(T^{*}-\tau^{\prime}\right) /\left(T^{\prime}-\tau^{\prime}\right)<0$, and $\Delta T D T<0$. The total delay time decreases in this case with respect to the ideal optimum. Overall, the upper bound of demand change Type 2 has a delayed ending time as described by (25), but is with a reduced total delay time.

The upper bounds of the sub-optimality of QUEUE with respect to the off-line optimum are summarized as follows:

Upper bound Type 1: $\Delta T=0, \quad \Delta T D T=\frac{\left(s_{1}-s_{2}\right)\left[s_{1} g^{\min }-c B_{1}\right] B_{2}\left(T^{\prime}-\tau^{\prime}\right)^{2}}{4 c\left(s_{1} s_{2}-B_{1} s_{2}\right)}$

Upper bound Type 2: $\quad \Delta T=\frac{B_{1} s_{2}}{s_{1} s_{2}-B_{2} s_{1}}\left(T^{\prime}-\tau^{\prime}\right), \Delta T D T<0$

\section{QUEUE strategy for intersections with multi-phase signal plans}

Now we consider an oversaturated intersection controlled by signal plans with $N$ phases, where $N>2$ is an integer. Given the current detector data, the earliest ending time $T$ of the oversaturation period is ready to be calculated assuming constant arrival rate in the future. Without loss of generality, assume $s_{1} \geq s_{2} \geq \ldots \geq s_{N}$, then the basic QUEUE strategy at each cycle is

$$
g_{i}=\left\{\begin{array}{l}
g_{i}^{\max }, \text { if } g_{j}=g_{j}^{\min } \text { for any } j<i, \text { and } \frac{n_{i}}{\Delta n_{i}^{\min }}>T, i=1,2, \ldots N \\
g_{i}^{\min }, \text { else }
\end{array}\right.
$$

where $i$ and $j$ are indices for phases, $g_{i}{ }^{\max }$ and $g_{i}{ }^{\min }$ are the maximum and minimum values for green duration of phase $i, \Delta n_{i}^{\text {min }}$ is the minimum value of queue dissolving rate of phase $i$.

The optimality of (35) is proved in Appendix B.

Note that (35) gives only the fundamental equations describing the QUEUE strategy. Additional if-then rules that concern the prevention of waste of green time are needed. We can summarize from the previous sections the following rules (Table 1) to interpret the QUEUE strategy which also apply to the QUEUE strategy for multi-phase intersections.

Table 1. Summary of if-then rules for the QUEUE strategy

\section{Motivation}

\section{Description of the if-then rule}

Minimization of total delay time

If any prior phase $j(j<i)$ calls for a maximum green duration $\left(g_{j}=g_{j}^{\max }\right)$, then $g_{i}=g_{i}^{\min }$.

Minimization of the duration of the

If it is about to miss the earliest end of the oversaturation, then $g_{i}=g_{i}^{\min }$. oversaturation period 
Minimization of total delay time

Prevention of spillover

Prevention of green starvation
If neither of the above two conditions happens, then $g_{i}=g_{i}^{\max }$.

If the queue size of any conflicting phase $c$ breaks the compressed maximum queue constraint, then $g_{i}=g_{i}^{\max }-\Delta g$ and $g_{c}=g_{c}{ }^{\min }+\Delta g$, where $\Delta g$ is the additional green time needed to make the output flow of phase $h$ equals the corresponding inflow rate.

If the phase $i$ does not have enough inflow rate to be served in a maximum green duration, then $g_{i}=g_{i}{ }^{\max }-\Delta g$ and $g_{i+1}=g_{i+1}{ }^{\min }+\Delta g$, where $\Delta g$ is the unused green time in case when $g_{i}=g_{i}^{\max }$.

\section{Numerical examples}

\subsection{An intersection with piecewise constant demand}

The proposed QUEUE method was numerically tested in a hypothetical intersection system. Parameters for the system are listed as follows: $s_{1}=5400 \mathrm{veh} / \mathrm{h}, s_{2}=3600 \mathrm{veh} / \mathrm{h}, n_{1}{ }^{\max }=1200 \mathrm{veh}, n_{2}{ }^{\max }=1100 \mathrm{veh}, c=180 \mathrm{~s}, g^{\text {max }}=150 \mathrm{~s}$, and $g^{\mathrm{min}}=60 \mathrm{~s}$. The entire control duration is 3 hours, which contains 60 cycles $(180$ seconds a cycle). The demands for both approaches are assumed to change, if any, every 0.5 hours. Table 2 shows the demands used for the example.

Table 2. Tested demand profile

\begin{tabular}{ccccccc}
\hline Duration $(\mathrm{h})$ & $0 \sim 0.5$ & $0.5 \sim 1$ & $1 \sim 1.5$ & $1.5 \sim 2$ & $2 \sim 2.5$ & $2.5 \sim 3$ \\
\hline$d_{1}(\mathrm{veh} / \mathrm{h})$ & 5400 & 5400 & 3000 & 1600 & 1600 & 1600 \\
$d_{2}(\mathrm{veh} / \mathrm{h})$ & 1200 & 2400 & 1200 & 900 & 900 & 900 \\
$d_{1} / s_{1}+d_{2} / s_{2}$ & 1.333 & 1.667 & 0.889 & 0.546 & 0.546 & 0.546 \\
Division of the & Queuing period & Dissipation period & & \\
oversaturation period & & & & & \\
\hline
\end{tabular}

Three strategies, the ideal optimal strategy without maximum queue size constraints, the off-line optimal strategy with maximum queue size constraints, and the QUEUE strategy, were tested. Note again that the former two strategies are off-line strategies based on fully knowledge of the demand profiles, while the QUEUE strategy calculates on-line the green split based on current detected data cycle by cycle. The reserved queuing spaces are selected as $n_{1}{ }^{\text {res }}=300 \mathrm{veh}$ and $n_{2}{ }^{\text {res }}=300 \mathrm{veh}$.

First of all, the comparison of CSCs with different strategies is depicted in Fig. 14. The unconstrained optimal strategy is derived from the method introduced by Gazis (1964), which turns out a two-stage bang-bang control type with the switch time $\tau=1.5 \mathrm{~h}$. When the maximum queue constraints are taken into account, the off-line optimal control strategy and the QUEUE strategy allot some more green time to Approach 2 before $\tau$ to prevent the queue size on Approach 2 from exceeding the maximum value, and switch to the second stage some time later. The QUEUE strategy succeeds in eliminating both queues simultaneously at the earliest time $(T=2.5 \mathrm{~h})$ as the ideal optimal strategy does. Moreover, the QUEUE strategy almost matches the off-line optimal strategy with only $1.37 \%$ higher total delay time. 


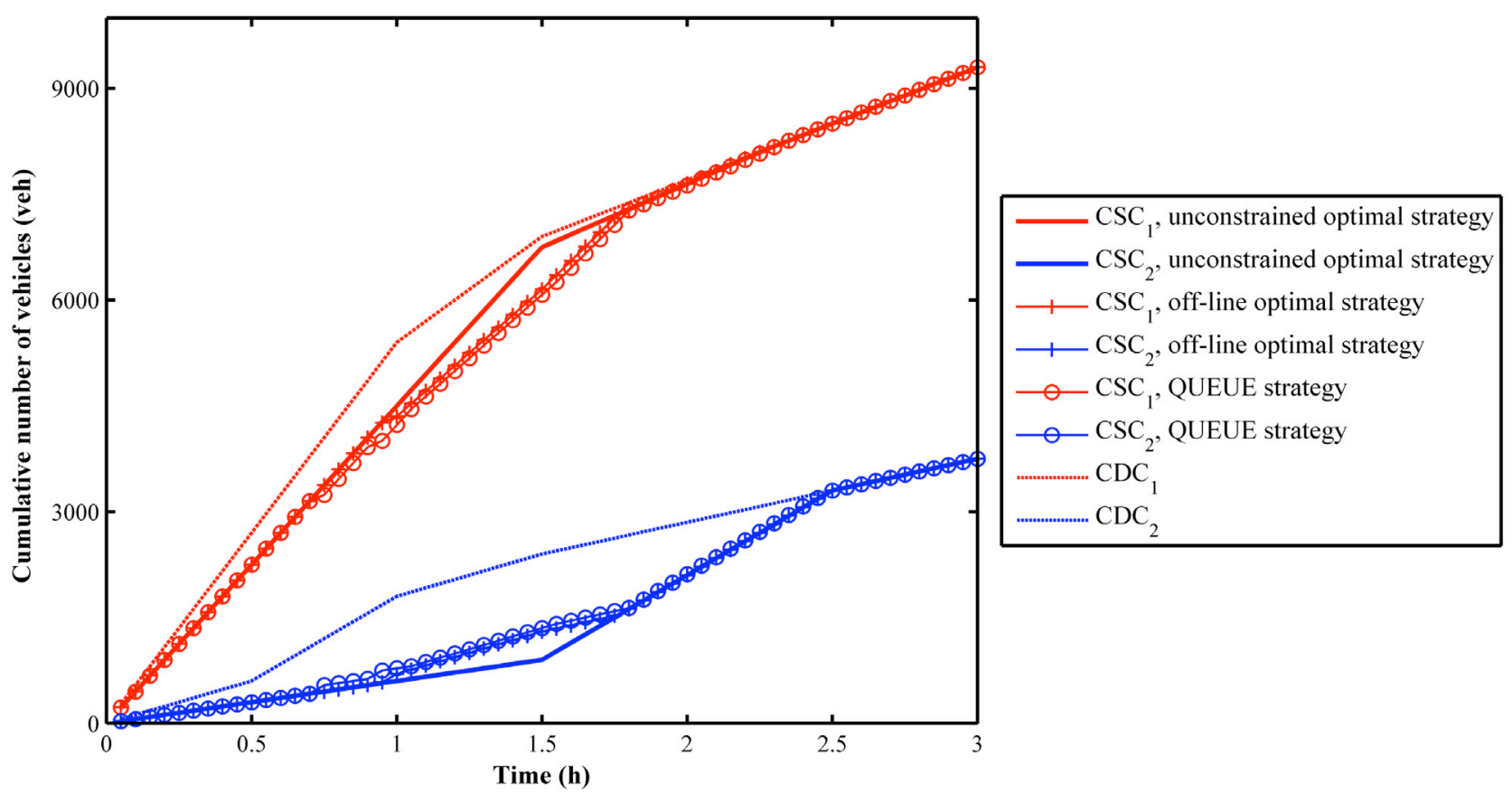

Fig. 14. Comparison of CSCs with different strategies

Fig. 15 shows the time-varying queue sizes on both roads with different strategies. It is clear that $n_{2}$ with the unconstrained optimal strategy exceeds the maximum value for about $1 \mathrm{~h}$. To conquer this, the trajectory of $n_{2}$ with the off-line optimal strategy is clipped by the maximum queue size line. As the overall queues growing is inevitable, the peak value of $n_{1}$ gets much closer to its maximum value with the off-line optimal strategy. It is exciting to find that the QUEUE strategy manages to keep queue sizes on both roads within the maximum values under this challenging condition.

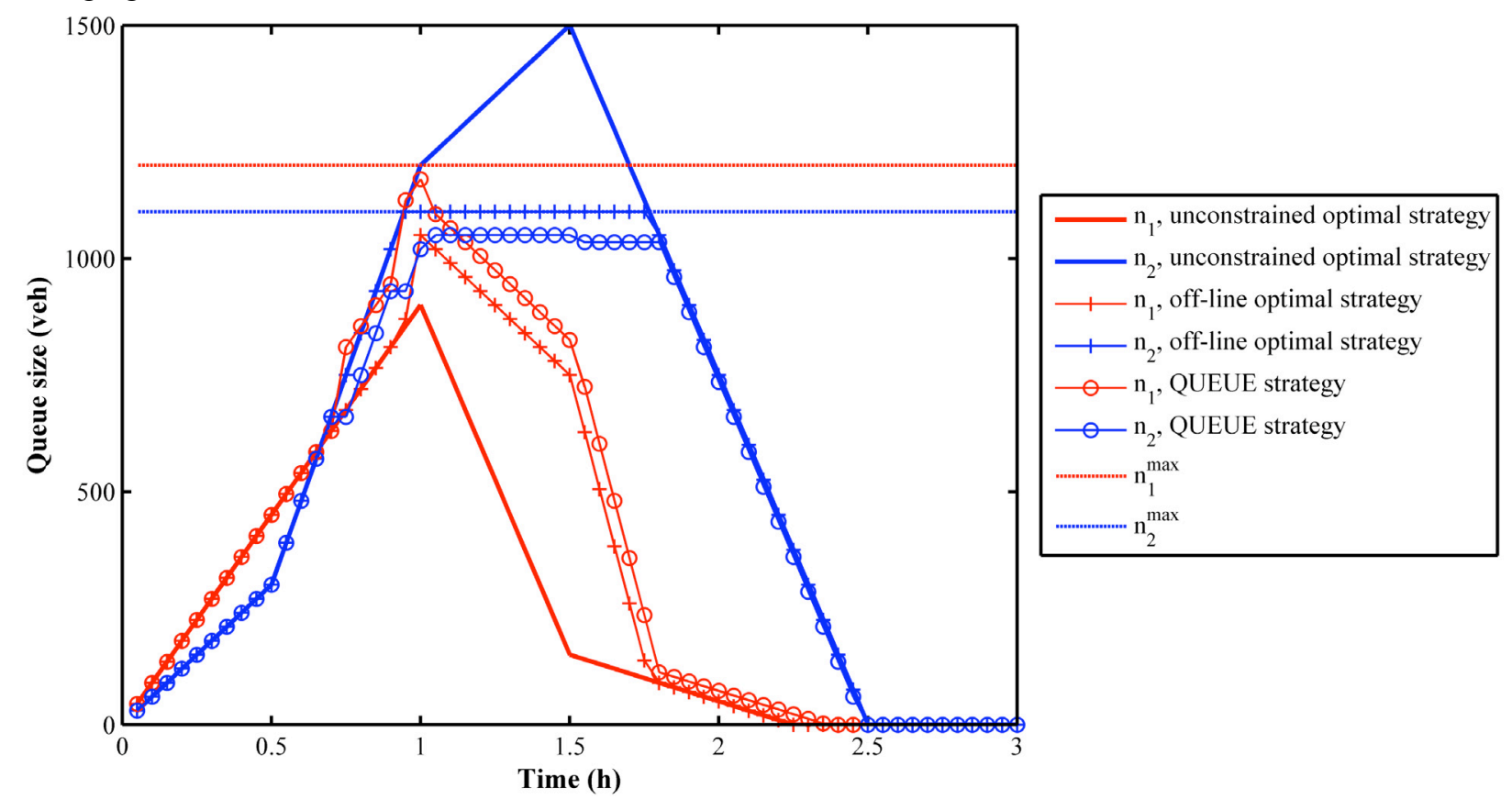

Fig. 15. Comparison of queue size trajectories with different strategies

Finally, the control trajectory of the QUEUE strategy is analyzed. In comparison with the two-stage ideal optimal strategy, the control trajectory of the QUEUE strategy usually has multiple stages. In this particular example, it has 8 stages in all (see Fig. 16). Stage 1 is the same with the optimal one, letting $g_{1}=g^{\max }$ at the beginning. At time $t=0.75 \mathrm{~h}, g_{1}$ is switched to $g^{\min }$ (Stage 2) to meet the compressed maximum queue constraints 
$\left[n_{1}{ }^{\text {max }}{ }_{-} n_{1}{ }^{\text {res }}, n_{2}{ }^{\text {max }}{ }_{-}{ }_{2}{ }^{\text {res }}\right]$. At time $t=0.8 \mathrm{~h}$, the compressed maximum queue constraints are broken and the final constraints $\left[n_{1}{ }^{\max }, n_{2}{ }^{\text {max }}\right]$ are considered. So a new sequence of $g_{1}=g^{\max }$ (Stage 3) and $g_{1}=g^{\min }$ (Stage 4) is set. At time $t=1 \mathrm{~h}$, the queuing period switches to the dissipation period as a result of demand drop, so $g_{1}=g^{\max }$ is set again (Stage 5). However, when the transition period together with a long queue on Approach 2 are detected ( $t=1.1 \mathrm{~h})$, the maximum feasible value $g^{\max }$ is replaced by the one determined by the demand $d_{2}$ so as to keep $n_{2}$ within the maximum constraint. As a consequence, Stage 6 and Stage 7 take place with respect to different demands. At time $t=1.85 \mathrm{~h}, g_{1}$ switches to $g^{\mathrm{min}}$ finally.

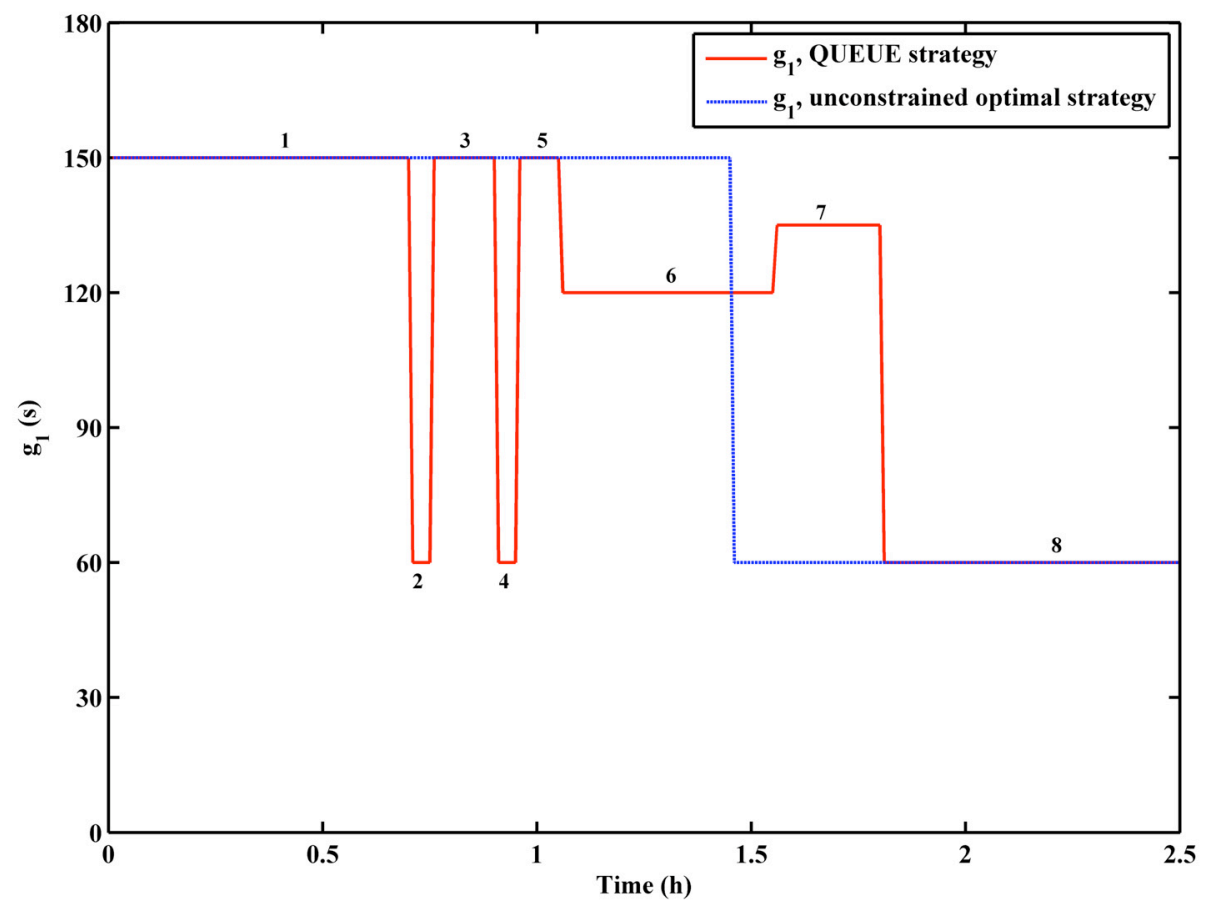

Fig. 16. Mutli-stage control trajectory of the QUEUE strategy

\subsection{An intersection with Poisson distributed random demand}

To test the performance of QUEUE strategy in the context of random demand fluctuation, the initial piecewise constant demands (Table 2) was replaced by Poisson distributed random values. For example, at each cycle ( $3 \mathrm{~min}$ ) during the time $t=0 \sim 0.5 \mathrm{~h}$, the actual arrival number of vehicle on road 1 was set a random value that follows Poisson distribution with the mean $\lambda=5400 \mathrm{veh} / \mathrm{h} * 3 \mathrm{~min}=270 \mathrm{veh}$. Demands for the rest of time were generated in the same manner (see Fig. 17). 


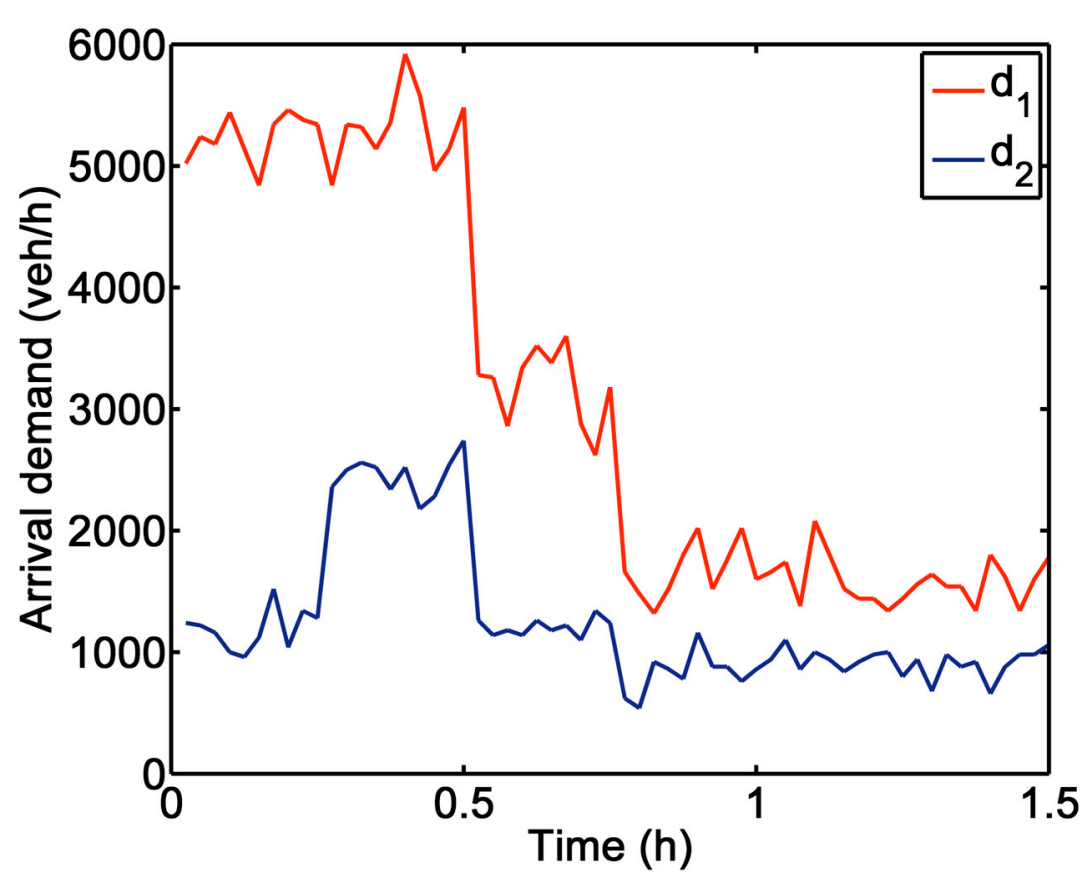

Fig. 17. Poisson distributed random demand profile

The consequent control result shows that the QUEUE strategy still did an excellent job. The queue size trajectories are shown in Fig. 18. The QUEUE strategy ended the oversaturation period 3 minute $(2.00 \%)$ later than the off-line optimum, and the difference of total delay time is $2.65 \%$ higher.

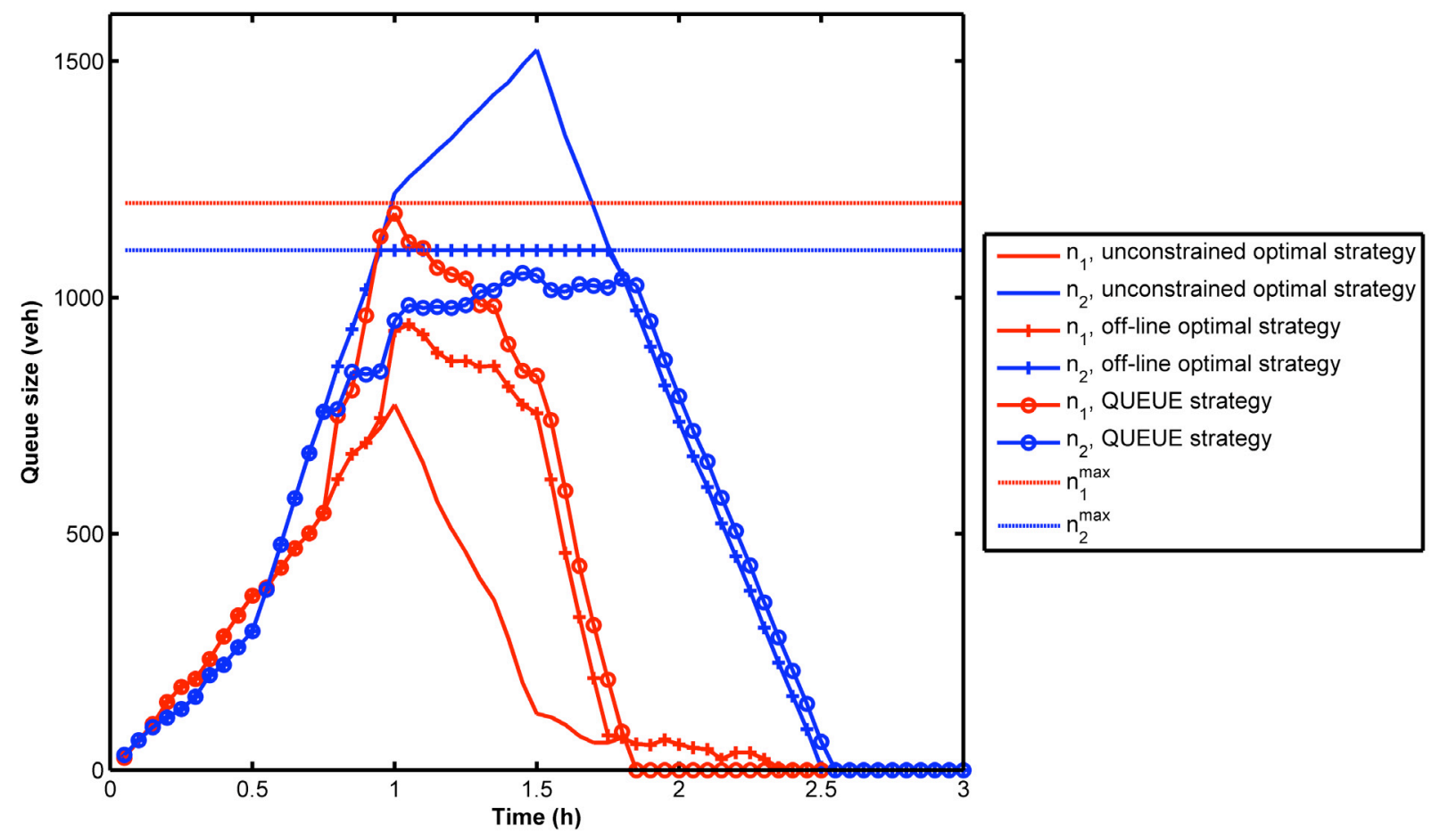

Fig. 18. Queue size trajectories with Poisson distributed demand

We then conduct a series of 20 tests, each with randomly generated demands followed the same Poisson distribution. The values of $\Delta T$ and $\triangle T D T$ in terms of percentage with respect to the off-line optimum are summarized in Table 4. The average values of oversaturation ending time difference $(\Delta T)$ and the difference of the total delay time $(\triangle T D T)$ are $4.18 \%$ and $0.91 \%$. The value of $\Delta T$ may go as high as $17.65 \%$, but it can be further 
improved by adopting filtering algorithms that eliminate the influence of high-frequency large-amplitude demand fluctuation. The values of $\triangle T D T$ never exceed $4 \%$, and are negative in several tests. The results demonstrate that the QUEUE strategy performs very close to the off-line optimal strategy, and it may further reduce the total delay time comparing to the off-line optimal strategy at the cost of some delayed ending time of the oversaturation period.

Table 4. Results of tests with random demand that follows the same Poisson distribution

\begin{tabular}{cccccc}
\hline Test & $\Delta T(\%)$ & $\begin{array}{c}\Delta T D T \\
(\%)\end{array}$ & Test & $\Delta T(\%)$ & $\begin{array}{c}\Delta T D T \\
(\%)\end{array}$ \\
\hline 1 & $2.00 \%$ & $2.65 \%$ & 11 & $2.04 \%$ & $-0.43 \%$ \\
2 & $17.65 \%$ & $3.03 \%$ & 12 & $1.96 \%$ & $-1.28 \%$ \\
3 & $1.96 \%$ & $0.31 \%$ & 13 & $2.00 \%$ & $0.34 \%$ \\
4 & $2.00 \%$ & $0.70 \%$ & 14 & $17.65 \%$ & $3.23 \%$ \\
5 & $2.00 \%$ & $2.10 \%$ & 15 & $4.08 \%$ & $2.10 \%$ \\
6 & $2.04 \%$ & $-0.25 \%$ & 16 & $2.04 \%$ & $-0.94 \%$ \\
7 & $4.17 \%$ & $1.43 \%$ & 17 & $4.08 \%$ & $1.89 \%$ \\
8 & $1.89 \%$ & $-0.85 \%$ & 18 & $3.92 \%$ & $-1.02 \%$ \\
9 & $2.04 \%$ & $3.04 \%$ & 19 & $2.04 \%$ & $1.56 \%$ \\
10 & $4.08 \%$ & $1.68 \%$ & 20 & $4.00 \%$ & $-1.01 \%$ \\
& & & Average & $4.18 \%$ & $\mathbf{0 . 9 1 \%}$ \\
\hline
\end{tabular}

\section{Discussion and concluding remarks}

In this paper, we propose a queue-based on-line quasi-optimal feedback control (QUEUE) strategy for an isolated oversaturated intersection. The description and analysis of the QUEUE strategy for an intersection with two-phase signal plans are exhibited in detail; the extension to a multi-phase intersection is briefly introduced as well.

It is inspiring to find that the QUEUE strategy can approximate the off-line optimum in an on-line feedback manner which means that it can be readily used in practice. The outstanding performances of the QUEUE strategy are concluded as follows:

1. For constant demand, it is proved to perfectly match the off-line optimum.

2. For piece-wise constant demand, the upper bounds of the sub-optimality can be quantitatively calculated, and lie in a reasonable range.

3. For Poisson distributed random demand, it is shown in numerical examples that the average sub-optimality is less than $5 \%$ with respect to the off-line optimum.

The QUEUE strategy for one isolated intersection is the first step to deal oversaturation. If more than one oversaturated intersections are located adjacently, the QUEUE strategy can also work with some necessary extension. The main idea is that the QUEUE strategy will always run locally at each intersection, i.e., a decentralized strategy. The basic control logic is the same as proposed in this paper. Most of the time, the QUEUE strategy optimally allocates the green splits of a particular intersection based on the queue sizes and arrival flow rates on its own approaches. Considering the interaction of two adjacent intersections, efforts must be made to prevent the queue spillover to upstream intersection as well as the waste of green time caused by insufficient supply to downstream intersection. To these ends, queue sizes of adjacent intersections should also be measured and considered in the determination of the signal timing for the subject intersection, and proper threshold values of queue sizes should be set to trigger special tactics such as upstream gating. We should note that, however, such 
extension is not straightforward. Solid theoretical analysis and comprehensive numerical testing are needed to develop the extended QUEUE strategy and test its optimality (or sub-optimality). We leave these topics for future research.

\section{Appendix A. Proof of optimality in case of constant arrival demand}

With constant arrival demand, we are to prove the optimality of the basic equation (12) of the QUEUE strategy in case when $s_{1} \mathrm{gm} / \mathrm{c} \geq B_{1}$ in detail, on the basis of which the optimality of the ameliorations for the two special cases and the final equation (15) of the QUEUE strategy could be easily proved. We start with several lemmas.

Lemma A1. Assume that the arrival flow rates are constant and $s_{1} g^{\min } / \mathrm{c} \geq B_{1}$, then, the feedback strategy (12) has the same structure (either one-stage or two-stage) with the ideal optimal one.

Proof. As mentioned by Gazis (1964), the existence of the two-stage optimal solution is based on the following assumption:

$$
g^{\min }<\frac{c\left(A_{2} B_{1}-A_{1} B_{2}+A_{1} s_{2}\right)}{A_{1} s_{2}+A_{2} s_{1}}<g^{\max }
$$

With this assumption, the optimal strategy is letting $g_{1}=g^{\max }$ in the first stage and $g_{1}=g^{\min }$ in the second stage. We now check whether the feedback strategy (12) performs in the same way.

Note that $\left[n_{1}{ }^{0}, n_{2}{ }^{0}\right]=\left[A_{1}, A_{2}\right]$, (A1) is equivalent to

$$
\left(\frac{\Delta n_{1}}{\Delta n_{2}}\right)^{\min }<\frac{n_{1}^{0}}{n_{2}^{0}}<\left(\frac{\Delta n_{1}}{\Delta n_{2}}\right)^{\max }
$$

Consequently, $g_{1}=g^{\max }$ is set at the very beginning. As long as $n_{1} / n_{2}>\left(\Delta n_{1} / \Delta n_{2}\right)^{\min }$ holds, the derivative of $n_{1} / n_{2}$ is written as

$$
\frac{d}{d t}\left(\frac{n_{1}}{n_{2}}\right)=\frac{n_{2} \cdot \frac{d}{d t} n_{1}-n_{1} \cdot \frac{d}{d t} n_{2}}{n_{2}^{2}}=\frac{n_{2}\left(B_{1}-s_{1} g^{\max } / c\right)-n_{1}\left(B_{2}-s_{2} g^{\min } / c\right)}{n_{2}^{2}}
$$

As long as $n_{1} / n_{2}<\left(\Delta n_{1} / \Delta n_{2}\right)^{\max }$ holds,

$$
\frac{d}{d t}\left(\frac{n_{1}}{n_{2}}\right)=\frac{n_{2}\left(B_{1}-s_{1} g^{\max } / c\right)-n_{1}\left(B_{2}-s_{2} g^{\min } / c\right)}{n_{2}^{2}}<0
$$

Note that $\frac{d}{d t}\left(\frac{n_{1}}{n_{2}}\right)$ is negative, so $n_{1} / n_{2}$ will decrease continuously until the queue state $\left[n_{1}, n_{2}\right]$ reaches any boundary. There are four boundaries in total, i.e., the upper boundary $n_{1} / n_{2}=\left(\Delta n_{1} / \Delta n_{2}\right)^{\max }$, the lower boundary $n_{1} / n_{2}=\left(\Delta n_{1} / \Delta n_{2}\right)^{\min }$, the horizontal axis $n_{1} / n_{2}=0$ and the vertical axis $n_{1} / n_{2}= \pm \infty$. As assumed,

$$
0<\left(\frac{\Delta n_{1}}{\Delta n_{2}}\right)^{\min }<\frac{n_{1}^{0}}{n_{2}^{0}}<\left(\frac{\Delta n_{1}}{\Delta n_{2}}\right)^{\max }<\infty,
$$

so the first boundary that $\left[n_{1}, n_{2}\right]$ reaches is the lower boundary $n_{1} / n_{2}=\left(\Delta n_{1} / \Delta n_{2}\right)^{\min }$. And it is obvious from (12) that 
as long as $n_{1} / n_{2}=\left(\Delta n_{1} / \Delta n_{2}\right)^{\mathrm{min}}$, the equation will hold until $\left[n_{1}, n_{2}\right]=[0,0]$. In other words, two stages constitute the feedback strategy (12), which are $g_{1}=g^{\max }$ in the first stage and $g_{1}=g^{\min }$ in the second stage. It exhibits the same two-stage structure as the optimal strategy does.

We need also discuss conditions in which (A1)-(A2) do not hold. Except for the attractable region of the origin, there are another two regions in the first quadrant (Fig. A1), expressed by the following inequalities, respectively.

$$
\begin{aligned}
& 0<\frac{n_{1}^{0}}{n_{2}^{0}} \leq\left(\frac{\Delta n_{1}}{\Delta n_{2}}\right)^{\min } \\
& \left(\frac{\Delta n_{1}}{\Delta n_{2}}\right)^{\max } \leq \frac{n_{1}^{0}}{n_{2}^{0}}<\infty
\end{aligned}
$$

If (A6) holds, it is proved by Gazis that letting $g_{1}=g^{\min }$ till both queues are dissolved is the optimal strategy. With the feedback strategy (12), $g_{1}=g^{\min }$ is set at the very beginning, and as long as $n_{1} / n_{2}<\left(\Delta n_{1} / \Delta n_{2}\right)^{\min }$, we have

$$
\frac{d}{d t}\left(\frac{n_{1}}{n_{2}}\right)=\frac{n_{2} \cdot \frac{d}{d t} n_{1}-n_{1} \cdot \frac{d}{d t} n_{2}}{n_{2}^{2}}=\frac{n_{2}\left(B_{1}-s_{1} g^{\min } / c\right)-n_{1}\left(B_{2}-s_{2} g^{\max } / c\right)}{n_{2}^{2}}<0
$$

So $n_{1} / n_{2}$ will decrease continuously to 0 and then $-\infty$. This is shown in Fig. A1 by the red arrow crosses the horizontal axis and then reaches the vertical axis. Note that the actual trajectory should never go out of the first quadrant, but is bounded by the axes. However, it is depicted by a straight line here to illustrate that the control strategy is letting $g_{1}=g^{\min }$ continuously without any switch, which coincides with the optimal one.

If (A7) holds, it can be proved in the same way that the feedback strategy (12) coincides with the optimal one, which is letting $g_{1}=g^{\max }$ till both queues are dissolved. This completes the proof of Lemma A1.

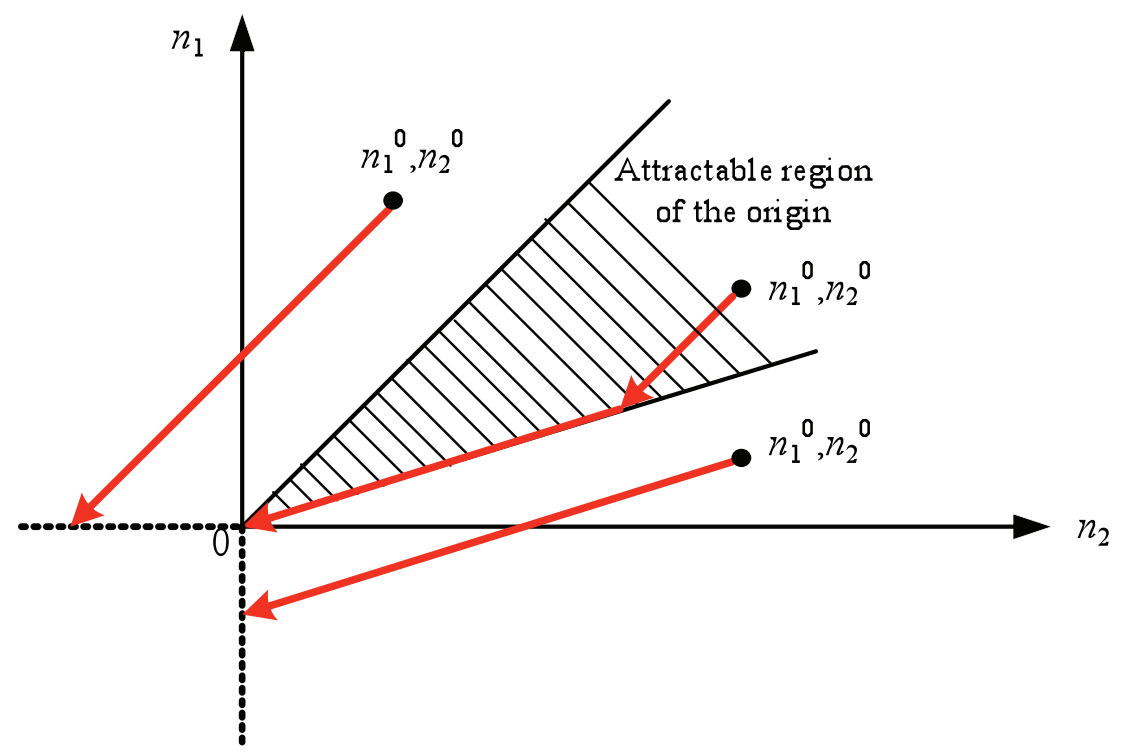

Fig. A1 Schematic optimal trajectories starting from different regions

Lemma A2. Assume that the arrival flow rates are constant and the two-stage optimal strategy shown in Fig. 2 exists, then, the feedback strategy (12) switches to the second stage at the same time as the optimal one does shown in (8). 
Proof. According to Lemma 1, the control strategy derived from the feedback strategy (12) is letting $g_{1}=g^{\max }$ in the first stage and $g_{1}=g^{\text {min }}$ in the second stage. With the assumption that arrival flow rates are constant, the derivative of either $n_{1}$ or $n_{2}$ is constant. So either $n_{1}$ or $n_{2}$ is a linear function of time $t$ at the first stage. Denote the corresponding switch time by $\tau_{0}$. Then, at time $\tau_{0}$, we have

$$
\frac{n_{1}^{0}+\tau_{0} \cdot\left(B_{1}-s_{1} g^{\max } / c\right)}{n_{2}^{0}+\tau_{0} \cdot\left(B_{2}-s_{2}\left(1-g^{\max } / c\right)\right)}=\frac{B_{1}-s_{1} g^{\min } / c}{B_{2}-s_{2}\left(1-g^{\min } / c\right)}
$$

Note that $\left[n_{1}{ }^{0}, n_{2}{ }^{0}\right]=\left[A_{1}, A_{2}\right]$, the value of $\tau_{0}$ is easily calculated from (A9).

$$
\tau_{0}=\frac{A_{2} B_{1} c-A_{1} B_{2} c+A_{1} s_{2}\left(c-g^{\min }\right)-A_{2} s_{1} g^{\min }}{\left(s_{1} s_{2}-B_{1} s_{2}-B_{2} s_{1}\right)\left(g^{\max }-g^{\mathrm{min}}\right)}
$$

Compare (A10) with (8), and $\tau_{0}=\tau^{*}$ is proved.

Then, combining Lemma A1 and Lemma A2 immediately gives the following theorem.

Theorem A1. Assume that the arrival flow rates are constant and $s_{1} g^{\mathrm{min}} / \mathrm{c} \geq B_{1}$, then, (12) gives the ideal optimal strategy for the dissipation period.

\section{Appendix B. Proof of optimality for multi-phase intersection}

We show the optimality of (35) by contradiction. If we put the control solution derived from (35) at each cycle together, it turns out an $N \times K$ matrix $\mathbf{G}$, where $N$ is the number of signal phases and $K=T / c$ is the least total number of cycles needed to end the oversaturation period.

$$
\mathbf{G}=\left[\begin{array}{cccc}
g_{1,1} & g_{1,2} & \ldots & g_{1, K} \\
g_{2,1} & g_{2,2} & \ldots & g_{2, K} \\
\cdots & \ldots & \ldots & \ldots \\
g_{N, 1} & g_{N, 2} & \ldots & g_{N, K}
\end{array}\right]
$$

where $g_{i, x}$ is the green duration of phase $i$ at cycle $x, i=1,2, \ldots, N, x=1,2, \ldots, K$.

It is easy to find that the summation of each row or each column of $\mathbf{G}$ is constant,

$$
\sum_{i=1}^{N} g_{i, k}=c, \sum_{k=1}^{K} g_{i, k}=\frac{C D C_{i, K}}{s_{i}}
$$

Consider another solution, an $N \times K$ matrix $\mathbf{G}_{\text {a }}$ which is different from $\mathbf{G}$, that also ends the oversaturation period at the earliest time, we are to prove that $\mathbf{G}_{\mathrm{a}}$ is less optimal in terms of the total delay time in the following.

Since (B2) still holds for $\mathbf{G}_{\mathbf{a}}$, there must be at least four pairs of different elements between the two matrices. For example,

$$
\mathbf{G}=\left[\begin{array}{ccccc}
. . & \ldots & \ldots & \ldots & \ldots \\
. . & g_{i, x} & \ldots & g_{i, y} & \ldots \\
. . & \ldots & \ldots & \ldots & \ldots \\
. . & g_{j, x} & \ldots & g_{j, y} & \ldots \\
. . & \ldots & \ldots & \ldots & \ldots
\end{array}\right] \leftrightarrow \mathbf{G}_{\mathrm{a}}=\left[\begin{array}{ccccc}
. . & \ldots & \ldots & \ldots & \ldots \\
. & g_{i, x}^{a} & \ldots & g_{i, y}^{a} & \ldots \\
. . & \ldots & \ldots & \ldots & \ldots \\
. . & g_{j, x}^{a} & \ldots & g_{j, y}^{a} & \ldots \\
. . & \ldots & \ldots & \ldots & \ldots
\end{array}\right]
$$

where $g_{i, x^{-}} g_{i, x}^{\mathrm{a}}=g_{i, y^{-}}^{\mathrm{a}} g_{i, y^{\prime}}=g_{j, x^{-}}^{\mathrm{a}} g_{j, x}=g_{j, y^{-}} g_{j, y^{\mathrm{a}}}^{\mathrm{a}}=\Delta g \neq 0$. 
Lemma B1. If $i<j$ and $x<y$ as shown in (B3), then $g_{i, x}=g_{i}^{\max }, g_{i, y}=g_{i}^{\min }, g_{j, x}=g_{j}^{\min }, g_{j, y}=g_{j}^{\max }$, and $\Delta g>0$.

Proof. We know form (35) that $g_{i, x}=g_{i}^{\max }$ or $g_{i}^{\min }$. If $g_{i, x}=g_{i}^{\max }$, then $g_{i, x} \geq g_{i, x}^{\mathrm{a}}$, so $\Delta g>0$. In such a case, $g_{i, y}=g_{i}^{\min }$ must hold; otherwise, if $g_{i, y}=g_{i}^{\max }, g_{i, y}^{\mathrm{a}}=g_{i, y}+\Delta g$ will break the maximum green time constraint. Similarly, we get $g_{j, x}=g_{j}^{\min }$ and $g_{j, y}=g_{j}^{\max }$. On the other hand, if $g_{i, x}=g_{i}^{\min }$, we get $g_{i, y}=g_{i}^{\max }, g_{j, x}=g_{j}^{\max }$ and $g_{j, y}=g_{j}^{\min }$.

Overall, there are two sets of feasible values,

$$
g_{i, x}=g_{i}^{\max }, g_{i, y}=g_{i}^{\min }, g_{j, x}=g_{j}^{\min }, g_{j, y}=g_{j}^{\max } \text {, and } \Delta g>0 \text {, }
$$

or

$$
g_{i, x}=g_{i}^{\min }, g_{i, y}=g_{i}^{\max }, g_{j, x}=g_{j}^{\max }, g_{j, y}=g_{j}^{\min } \text {, and } \Delta g<0 \text {. }
$$

However, if we assume that (B5) is the case, since $g_{i, y}=g_{i}^{\max }$, it follows from (B1) that

$$
\frac{n_{i, y}}{\Delta n_{i}^{\min }}>T
$$

Note that the arrival flow rates are constant, so the value of $\Delta n_{i}{ }^{\min }$ is also constant. Moreover, the queue length in the dissipation period is decreasing, so $n_{i, x}>n_{i, y}$, and

$$
\frac{n_{i, x}}{\Delta n_{i}^{\min }}>\frac{n_{i, y}}{\Delta n_{i}^{\min }}>T
$$

Since $g_{i, x}=g_{i}^{\min }$, comparing (35) and (B7) reveals that there exists a phase $h$ that satisfies $h<i<j$, and $g_{h, x}=g_{h}{ }^{\max }$.

Then we get $g_{j, x}=g_{j}^{\text {min }}$ from (35), which is in contradiction to (B5).

It is now proved that (B5) does not coincide with the initial strategy (B1). So (B4) is the only possibility.

Lemma B2. The total delay time applying $\mathbf{G}_{\mathrm{a}}$ is greater than that applying $\mathbf{G}$.

Proof. First, the following recursive equations are derived regarding the conservation of vehicles

$$
\begin{aligned}
n_{i, k} & =n_{i, k-1}+d_{i, k}-s_{i} \cdot g_{i, k} / c \\
& =n_{i, k-2}+d_{i, k-1}-s_{i} \cdot g_{i, k-1} / c+d_{i, k}-s_{i} \cdot g_{i, k} / c \\
& \ldots \\
& =n_{i, 0}+\sum_{m=1}^{k} d_{i, m}+s_{i} / c \cdot \sum_{m=1}^{k} g_{i, m}
\end{aligned}
$$

where $n_{i, 0}$ is the initial queue size of phase $i$.

So the total delay time is

$$
\begin{aligned}
T D T & =\sum_{i=1}^{N} \sum_{k=1}^{K} n_{i, k} \\
& =\sum_{i=1}^{N} \sum_{k=1}^{K}\left(n_{i, 0}+\sum_{m=1}^{k} d_{i, m}\right)+\sum_{i=1}^{N}\left[s_{i} / c \cdot \sum_{k=1}^{K}\left(\sum_{k=1}^{k} g_{i, m}\right)\right] \\
& =\sum_{i=1}^{N} \sum_{k=1}^{K}\left(n_{i, 0}+\sum_{m=1}^{k} d_{i, m}\right)+\sum_{i=1}^{N}\left[s_{i} / c \cdot \sum_{k=1}^{K}\left((K+1-k) g_{i, k}\right)\right]
\end{aligned}
$$

where the first component is a constant, and the second one is a function of the controlled green splits. 
Then, the difference of total delay time between the two solutions $\mathbf{G}$ and $\mathbf{G}_{\mathrm{a}}$ is

$$
\begin{aligned}
\Delta T D T= & T D T\left(\mathbf{G}_{\mathrm{a}}\right)-T D T(\mathbf{G}) \\
= & \sum_{i=1}^{N}\left[s_{i} / c \cdot \sum_{k=1}^{K}\left((K+1-k) g_{i, k}^{\mathrm{a}}\right)\right]-\sum_{i=1}^{N}\left[f_{i} / c \cdot \sum_{k=1}^{K}\left((K+1-k) g_{i, k}\right)\right] \\
= & \left.s_{i} / c \cdot[K+1-x)\left(g_{i, x}^{\mathrm{a}}-g_{i, x}\right)+(K+1-y)\left(g_{i, y}^{\mathrm{a}}-g_{i, y}\right)\right] \\
& \left.+s_{j} / c \cdot[K+1-x)\left(g_{j, x}^{\mathrm{a}}-g_{j, x}\right)+(K+1-y)\left(g_{j, y}^{\mathrm{a}}-g_{j, y}\right)\right] \\
= & s_{i} / c \cdot(y-x) \Delta g+s_{j} / c \cdot(x-y) g \\
= & (y-x)\left(s_{i}-s_{j}\right) \Delta g / c
\end{aligned}
$$

Here $y>x$ and $s_{i}>s_{j}$ are assumed, and we know from Lemma B1 that $\Delta g>0$. As a consequence, $\triangle T D T>0$. In other words, the total delay time applying $\mathbf{G}_{\mathbf{a}}$ is greater than that applying $\mathbf{G}$.

We can prove similarly that any other solution that ends the oversaturation period at the earliest time but has more than four different elements from $\mathbf{G}$ is less optimal in terms of the total delay time.

Finally, we get the following theorem.

Theorem B1 Assume that the arrival flow rates are constant, then, (35) gives the ideal optimal multi-phase control strategy for the dissipation period.

Acknowledgement: This research was funded partially by the National Science Foundation of China under Grant \#51328801 and Grant \#51278021. The views are those of the authors alone.

\section{References}

Aboudolas, K., Papageorgiou M. and Kosmatopoulos E., 2009. Store-and-forward based methods for the signal control problem in large-scale congested urban road networks. Transportation Research Part C 17(2), 163-174.

Aboudolas, K., Papageorgiou, M., Kouvelas, A. and Kosmatopoulos, E., 2010. A rolling horizon quadratic programming approach to signal control problem in large scale congested urban road networks. Transportation Research Part C, 18(5), 680-694.

Chang, T.H. and Lin, J.T., 2000. Optimal signal timing for an oversaturated intersection.

Transportation Research Part B 34(6), 471-491.

Daganzo, C., 2007. Urban gridlock: Macroscopic modeling and mitigation approaches. Transportation Research Part B, 41(1), 49-62.

Diakaki, C., Papageorgiou, M. and Aboudolas, K., 2002. A multivariable regulator approach to traffic responsive networkwide signal control. Control Engineering Practice 10(2), 183-195

Gazis, D.C., 1964. Optimum control of a system of oversaturated intersections. Operations Research 12(6), 815-831.

Gordon, R.L., 1969. A Technique for Control of Traffic at Critical Intersections. Transportation Science 3(4), 279-288.

Guardabassi, G., Locatelli, A. and Papageorgiou, M., 1984. A note on the optimal control of an oversaturated intersection. Transportation Research Part B 18(2), 111-113. 
Ioslovich, I., Haddad, J., Gutman, P. and Mahalel, D., 2011. Optimal traffic control synthesis for an isolated intersection. Control Engineering Practice 19(8), 900-911.

Lertworawanich, P., Kuwahara, M. and Miska, M., 2011. A new multiobjective signal optimization for oversaturated networks. IEEE Transactions on Intelligent Transportation Systems 12(4), 967-976.

Lieberman, E.B., Chang, J.N. and Prassas, E.S., 2000. Formulation of real-time control policy for oversaturated arterials. Transportation Research Record 1727, 77-88.

Lin, W., Lo, H.K. and Xiao, L., 2011. A quasi-dynamic robust control scheme for signalized intersections. Journal of Intelligent Transportation Systems 15(4): 223-233.

Liu, H.X., Wu, X., Ma, W. and Hu, H., 2009. Real-time queue length estimation for congested signalized intersections. Transportation Research Part C 17(4), 412-427.

Liu, Y. and Chang, G., 2011. An arterial signal optimization model for intersections experiencing queue spillback and lane blockage. Transportation Research Part C 19(1), 130-144.

Michalopoulos, P.G. and Stephanopoulos, G., 1977a. Oversaturated signal systems with queue length constraints-I: Single intersection. Transportation Research 11(6), 413-421.

Michalopoulos, P.G. and Stephanopoulos, G., 1977b. Oversaturated signal systems with queue length constraints - II: Systems of intersections. Transportation Research 11(6), 423-428.

Park, B., Messer, C.J. and Urbanik, T., 2000. Enhanced genetic algorithm for signal-timing optimization of oversaturated intersections. Transportation Research Record 1727, 32-41.

Varaiya, P., 2013. Max pressure control of a network of signalized intersections. Transportation Research Part C 36: 177-195.

$\mathrm{Wu}, \mathrm{X}$., Liu, H.X., and Gettman, D., 2010. Identification of oversaturated intersections using high-resolution traffic signal data. Transportation Research Part C 18(4), 626-638.

Zou, B., Hu, J. and Zhang, Y., 2012. The optimal discretized timing plan for individual oversaturated intersections. In: 15th International IEEE Conference on Intelligent Transportation Systems, Anchorage, Alaska, 1656-1660. 